\title{
SECOND ORDER OPTIMALITY CONDITIONS FOR SEMILINEAR ELLIPTIC CONTROL PROBLEMS WITH FINITELY MANY STATE CONSTRAINTS*
}

\author{
EDUARDO CASAS $^{\dagger}$ AND MARIANO MATEOS ${ }^{\ddagger}$
}

\begin{abstract}
This paper deals with necessary and sufficient optimality conditions for control problems governed by semilinear elliptic partial differential equations with finitely many equality and inequality state constraints. Some recent results on this topic for optimal control problems based upon results for abstract optimization problems are compared with some new results using methods adapted to the control problems. Meanwhile, the Lagrangian formulation is followed to provide the optimality conditions in the first case; the Lagrangian and Hamiltonian functions are used in the second statement. Finally, we prove the equivalence of both formulations.
\end{abstract}

Key words. necessary and sufficient optimality conditions, control of elliptic equations, state constraints

AMS subject classifications. 49K20, 35J25

PII. S0363012900382011

1. Introduction. The first goal of this paper is to provide some new second order optimality conditions for control problems of semilinear elliptic partial differential equations with finitely many state constraints. These conditions involve the Lagrangian and the Hamiltonian functions. Therefore, they are not a consequence of some abstract theorems in optimization theory but are proved by using arguments valid only in the framework of control theory. The second goal is to compare these conditions with those obtained recently for the same type of problems by using theorems for abstract optimization in infinite-dimensional spaces.

While there exists a very extensive literature about first order optimality conditions for control problems of partial differential equations, only a few papers are devoted to second order conditions. However, some progress has been made in the last few years. Most of the papers have been devoted to the study of sufficient second order optimality conditions; see Goldberg and Tröltzsch [13], Casas, Tröltzsch, and Unger [9], [10], Raymond and Tröltzsch [20]. Such sufficient optimality conditions are useful for carrying out the numerical analysis of a control problem, for obtaining error estimates in the numerical discretization, and for analyzing the sequential quadratic programming algorithms applied to control problems. However, we also have to study the second order necessary conditions and compare them with the sufficient ones in order to check if there is a reasonable gap between them. This was studied by Casas and Tröltzsch [7], [8] and Casas, Mateos, and Fernández [5] for some control problems. In the last papers, the authors proved the results by using some methods of abstract optimization theory and by stating some new results in this abstract framework. The gap between the established necessary and the sufficient conditions was very small.

*Received by the editors December 4, 2000; accepted for publication (in revised form) June 23, 2001; published electronically January 18, 2002. This research was partially supported by Dirección General de Enseñanza Superior e Investigación Científica (Spain).

http://www.siam.org/journals/sicon/40-5/38201.html

${ }_{\dagger}^{\dagger}$ Departamento de Matemática Aplicada y Ciencias de la Computación, E.T.S.I. Industriales y de Telecomunicación, Universidad de Cantabria, 39005 Santander, Spain (eduardo.casas@unican.es).

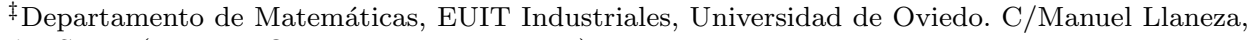
Gijón, Spain (mmateos@orion.ciencias.uniovi.es). 
Bonnans and Zidani [2] extended the results for finite-dimensional optimization problems to control problems by assuming that the second derivative with respect to the control of the Lagrangian function is a Legendre form. This is the natural way of doing such an extension, but the inconvenience is that the hypothesis about the Lagrangian function works in only a few cases. In this paper, instead of assuming that the second derivative of the Lagrangian function is a Legendre form, we assume a strict positivity condition on the second derivative of the Hamiltonian function with respect to the control, which is quite close to the necessary relaxed positivity.

The plan of the paper is as follows. In the next section, the control problem is formulated and some derivability results of the functionals are stated. In section 3, we reformulate the control problem as an infinite-dimensional optimization problem with constraints and we apply the second order conditions as deduced in [7] to our particular situation. Finally, in section 4 we deduce necessary and sufficient second order conditions involving the Lagrangian and the Hamiltonian functions and compare them with those established in section 3 .

2. The control problem. Let $\Omega$ be an open bounded set in $\mathbb{R}^{N}$ with a boundary $\Gamma$ of class $C^{1}$, and $A$ an elliptic operator of the form

$$
A y=-\sum_{i, j=1}^{N} \partial_{x_{j}}\left[a_{i j} \partial_{x_{i}} y\right]+a_{0} y,
$$

where the coefficients $a_{i j}$ belong to $C(\bar{\Omega})$ and satisfy

$$
m\|\xi\|^{2} \leq \sum_{i, j=1}^{N} a_{i j}(x) \xi_{i} \xi_{j} \leq M\|\xi\|^{2} \forall \xi \in \mathbb{R}^{N} \text { and } \forall x \in \Omega
$$

for some $m, M>0$ and $a_{0} \in L^{r}(\Omega)$ is not identically zero, with $r \geq N p /(N+p)$ for some $p>N$ fixed, $a_{0}(x) \geq 0$ in $\Omega$. Let $f$ and $L$ be Carathéodory functions $f: \Omega \times \mathbb{R}^{2} \rightarrow \mathbb{R}$ and $L: \Omega \times \mathbb{R}^{2} \longrightarrow \mathbb{R}, n_{e}$ and $n_{i}$ be nonnegative integers, and for every $1 \leq j \leq n_{e}+n_{i}$ let us consider a function $F_{j}: W^{1, p}(\Omega) \longrightarrow \mathbb{R}$.

The control problem is formulated as follows:

$$
(\mathbf{P})\left\{\begin{array}{l}
\text { Minimize } J(u)=\int_{\Omega} L\left(x, y_{u}(x), u(x)\right) d x, \\
u_{a}(x) \leq u(x) \leq u_{b}(x) \text { a.e. } x \in \Omega, \\
F_{j}\left(y_{u}\right)=0,1 \leq j \leq n_{e} \\
F_{j}\left(y_{u}\right) \leq 0, n_{e}+1 \leq j \leq n_{e}+n_{i}
\end{array}\right.
$$

where $y_{u}$ is the solution of

$$
\left\{\begin{aligned}
A y_{u} & =f\left(x, y_{u}, u\right) & & \text { in } \Omega \\
\partial_{n_{A}} y_{u} & =g & & \text { on } \Gamma
\end{aligned}\right.
$$

$g \in L^{p(1-1 / N)}(\Gamma)$ and $u_{a}, u_{b} \in L^{\infty}(\Omega), u_{a}(x) \leq u_{b}(x)$ for almost every (a.e.) $x \in \Omega$. Let us state the following assumptions on the functional $F_{j}, L$, and $f$.

(A1) $f$ is of class $C^{2}$ with respect to the second and third variables,

$$
f(\cdot, 0,0) \in L^{N p /(N+p)}(\Omega), \quad \frac{\partial f}{\partial y}(x, y, u) \leq 0,
$$

and for all $M>0$ there exists a constant $C_{f, M}>0$ such that

$$
\left|\frac{\partial f}{\partial y}(x, y, u)\right|+\left|\frac{\partial f}{\partial u}(x, y, u)\right|+\left|\frac{\partial^{2} f}{\partial y^{2}}(x, y, u)\right|+\left|\frac{\partial^{2} f}{\partial y \partial u}(x, y, u)\right|+\left|\frac{\partial^{2} f}{\partial u^{2}}(x, y, u)\right| \leq C_{f, M}
$$


for a.e. $x \in \Omega$ and $|y|,|u| \leq M$. Moreover, given $\rho>0$ arbitrary, for every $\varepsilon>0$ there exists $\delta>0$ such that for almost every point $x \in \Omega$ and $\left|y_{i}\right|,\left|u_{i}\right| \leq \rho, i=1,2$, we have

$$
\left|D_{(y, u)}^{2} f\left(x, y_{2}, u_{2}\right)-D_{(y, u)}^{2} f\left(x, y_{1}, u_{1}\right)\right|<\varepsilon \text { if }\left|y_{2}-y_{1}\right|<\delta,\left|u_{2}-u_{1}\right|<\delta,
$$

where $D_{(y, u)}^{2} f$ denotes the second derivative of $f$ with respect to $(y, u)$.

(A2) $L: \Omega \times \mathbb{R} \times \mathbb{R} \longrightarrow \mathbb{R}$ is of class $C^{2}$ with respect to the second and third variables, $|L(\cdot, 0,0)| \in L^{1}(\Omega)$, and for all $M>0$ there exists a constant $C_{M}>0$ and functions $\psi_{M} \in L^{N p /(N+p)}(\Omega)$ and $\psi_{M}^{*} \in L^{2}(\Omega)$ such that

$$
\left|\frac{\partial L}{\partial y}(x, y, u)\right| \leq \psi_{M}(x),\left|\frac{\partial L}{\partial u}(x, y, u)\right| \leq \psi_{M}^{*}(x)
$$

and

$$
\left|\frac{\partial^{2} L}{\partial y^{2}}(x, y, u)\right|+\left|\frac{\partial^{2} L}{\partial y \partial u}(x, y, u)\right|+\left|\frac{\partial^{2} L}{\partial u^{2}}(x, y, u)\right| \leq C_{M}
$$

for a.e. $x \in \Omega$ and $|y|,|u| \leq M$. Finally, given $\rho>0$ arbitrary, for every $\varepsilon>0$ there exists $\delta>0$ such that for almost every point $x \in \Omega$ and $\left|y_{i}\right|,\left|u_{i}\right| \leq \rho, i=1,2$, we have

$$
\left|D_{(y, u)}^{2} L\left(x, y_{2}, u_{2}\right)-D_{(y, u)}^{2} L\left(x, y_{1}, u_{1}\right)\right|<\varepsilon \text { if }\left|y_{2}-y_{1}\right|<\delta,\left|u_{2}-u_{1}\right|<\delta,
$$

where $D_{(y, u)}^{2} L$ denotes the second derivative of $L$ with respect to $(y, u)$.

(A3) For every $1 \leq j \leq n_{e}+n_{i}, F_{j}$ is of class $C^{1}$ in $W^{1, s}(\Omega)$ and of class $C^{2}$ in $W^{1, q}(\Omega)$, where $s \in\left[1, \frac{N}{N-1}\right), q \in\left[\max \left\{s, \frac{2 N}{N+2}\right\}, \frac{2 N}{N-2}\right)$, and $q \leq p$.

Remark 2.1. The continuity assumption on the coefficients $a_{i j}$ and the $C^{1}$ regularity of the boundary of the domain will allow us to consider integral state constraints involving the derivatives of the state. Nevertheless, if the coefficients $a_{i j}$ are only bounded and the boundary $\Gamma$ is Lipschitz, some results similar to those obtained here can be derived if the constraints do not involve the gradient of the state.

Let us show some examples of state constraints included in the previous formulation.

EXAmple 2.2. Integral constraints on the state. Given $g_{j}: \Omega \times \mathbb{R} \longrightarrow \mathbb{R}$, we define $F_{j}(y)=\int_{\Omega} g_{j}(x, y(x)) d x$. Assumption (A3) is satisfied if we make the following hypotheses: $g_{j}$ is of class $C^{2}$ with respect to the second variable and measurable with respect to the first one, $g_{j}(\cdot, 0) \in L^{1}(\Omega)$, and for every $M>0$ there exist $\psi_{M} \in L^{N s /([N+1] s-N)}(\Omega)$, for some $s<N /(N-1)$, and $\psi_{M}^{*} \in L^{\alpha}(\Omega)$, with $\alpha=1$ if $N<4$ and $\alpha>N / 4$ otherwise, such that for every $y, y_{1}, y_{2} \in[-M,+M]$ and almost every $x \in \Omega$

$$
\begin{gathered}
\left|\frac{\partial g_{j}}{\partial y}(x, y)\right| \leq \psi_{M}(x), \quad\left|\frac{\partial^{2} g_{j}}{\partial y^{2}}(x, y)\right| \leq \psi_{M}^{*}(x), \\
\forall \varepsilon>0 \exists \delta>0 \text { such that }\left|\frac{\partial^{2} g_{j}}{\partial y^{2}}\left(x, y_{2}\right)-\frac{\partial^{2} g_{j}}{\partial y^{2}}\left(x, y_{1}\right)\right| \leq \varepsilon \text { if }\left|y_{2}-y_{1}\right|<\delta .
\end{gathered}
$$

(A3) holds for $q=\min \{p, 2 N /(N-2)-\beta\}>N$ for some $\beta>0$ small enough.

EXAMPLE 2.3. Integral constraints on the derivatives of the state. Given $g_{j}$ : $\Omega \times \mathbb{R}^{N} \longrightarrow \mathbb{R}$, we now define $F_{j}(y)=\int_{\Omega} g_{j}(x, \nabla y(x)) d x$. Then assumption (A3) is 
fulfilled if $g_{j}$ is of class $C^{2}$ with respect to the second variable and measurable with respect to the first one, $g_{j}(\cdot, 0) \in L^{1}(\Omega)$, there exist $C>0, r<2 p / N, \psi \in L^{s^{\prime}}(\Omega)$ for some $s<N /(N-1)$, and $\psi^{*} \in L^{\alpha}(\Omega)$ with $\alpha>N / 2$, such that

$$
\left|\frac{\partial g_{j}}{\partial \eta}(x, \eta)\right| \leq \psi(x)+C|\eta|^{p(s-1) / s}, \quad\left|\frac{\partial^{2} g_{j}}{\partial \eta^{2}}(x, \eta)\right| \leq \psi^{*}(x)+C|\eta|^{r} \text {, for a.e. } x \in \Omega \text {, }
$$

and finally, for every $M>0$ and $\varepsilon>0$ there exists $\delta=\delta(\varepsilon, M)>0$ such that

$$
\left|\frac{\partial^{2} g_{j}}{\partial \eta^{2}}\left(x, \eta_{2}\right)-\frac{\partial^{2} g_{j}}{\partial \eta^{2}}\left(x, \eta_{1}\right)\right| \leq \varepsilon \text { if }\left|\eta_{2}-\eta_{1}\right|<\delta \text { and }\left|\eta_{1}\right|,\left|\eta_{2}\right| \leq M \text {, for a.e. } x \in \Omega \text {. }
$$

Once again, (A3) is fulfilled for $q=\min \{p, 2 N /(N-2)-\beta\}$ for $\beta>0$ small enough. The reader is referred to [5] for the study of this type of constraints.

The solution of (2.1) must be understood in a variational sense. Let us clarify this point. We define the variational form associated to the operator $A$ in the usual way:

$$
a(y, z)=\sum_{i, j=1}^{N} \int_{\Omega} a_{i j}(x) \partial_{x_{i}} y(x) \partial_{x_{j}} z(x) d x+\int_{\Omega} a_{0}(x) y(x) z(x) d x
$$

Then given $1<r<+\infty, \hat{f} \in\left(W^{1, r^{\prime}}(\Omega)\right)^{\prime}$, and $\hat{g} \in W^{-\frac{1}{r}, r}(\Gamma)$, we say that $y \in$ $W^{1, r}(\Omega)$ is a solution of

$$
\left\{\begin{array}{rll}
A y & =\hat{f} & \text { in } \Omega \\
\partial_{n_{A}} y=\hat{g} & \text { on } \Gamma
\end{array}\right.
$$

if

$$
a(y, z)=\langle\hat{f}, z\rangle_{\left(W^{1, r^{\prime}}(\Omega)\right)^{\prime} \times W^{1, r^{\prime}}(\Omega)}+\langle\hat{g}, \gamma z\rangle_{W^{-\frac{1}{r}, r}(\Gamma) \times W^{\frac{1}{r}, r^{\prime}}(\Gamma)} \forall z \in W^{1, r^{\prime}}(\Omega),
$$

where $\gamma: W^{1, r^{\prime}}(\Omega) \rightarrow W^{\frac{1}{r}, r^{\prime}}(\Gamma)$ is the trace operator. The following known result deals with the solvability of (2.2); see Mateos [17] for the details, as well as Morrey [19] and Troianiello [21].

Lemma 2.4. Let $1<r<+\infty, \hat{f} \in\left(W^{1, r^{\prime}}(\Omega)\right)^{\prime}$, and $\hat{g} \in W^{-\frac{1}{r}, r}(\Gamma)$. Then there exists a unique variational solution $y \in W^{1, r}(\Omega)$ of Neumann's problem (2.2). Moreover, the following estimate is satisfied:

$$
\|y\|_{W^{1, r}(\Omega)} \leq C\left(\|\hat{f}\|_{\left(W^{1, r^{\prime}}(\Omega)\right)^{\prime}}+\|\hat{g}\|_{W^{-\frac{1}{r}, r}(\Gamma)}\right),
$$

where $C>0$ is a constant only depending on $r$, the dimension $N$, the operator $A$, and the domain $\Omega$.

The semilinear case is a consequence of the previous lemma. In particular, $y_{u} \in$ $W^{1, p}(\Omega)$ is said to be a solution of (2.1) if it satisfies the above variational equation with $\hat{f}=f\left(\cdot, y_{u}, u\right) \in\left(W^{1, p^{\prime}}(\Omega)\right)^{\prime}$ and $\hat{g}=g \in L^{p(1-1 / N)}(\Gamma) \subset W^{-1 / p, p}(\Gamma)$. The next theorem states the existence and uniqueness of the solution of (2.1) as well as the differentiability of the relation between the control $u$ and the associated state $y_{u}$.

TheOREM 2.5. Suppose that (A1) holds. Then for every $u \in L^{\infty}(\Omega)$ there exists a unique solution $y_{u} \in W^{1, p}(\Omega)$ of the state equation (2.1) and

$$
\forall M>0 \exists C_{M}>0 \text { such that }\left\|y_{u}\right\|_{W^{1, p}(\Omega)} \leq C_{M} \text { if }\|u\|_{L^{\infty}(\Omega)} \leq M .
$$


The mapping $G: L^{\infty}(\Omega) \longrightarrow W^{1, p}(\Omega)$, defined by $G(u)=y_{u}$ is of class $C^{2}$ and for all $h, u \in L^{\infty}(\Omega), z_{h}=G^{\prime}(u) h$ is defined as the solution of

$$
\left\{\begin{aligned}
A z_{h} & =\frac{\partial f}{\partial y}\left(x, y_{u}, u\right) z_{h}+\frac{\partial f}{\partial u}\left(x, y_{u}, u\right) h & & \text { in } \Omega \\
\partial_{n_{A}} z_{h} & =0 & & \text { on } \Gamma .
\end{aligned}\right.
$$

Finally, for every $h_{1}, h_{2} \in L^{\infty}(\Omega), z_{h_{1} h_{2}}=G^{\prime \prime}(u) h_{1} h_{2}$ is the solution of

$$
\left\{\begin{aligned}
A z_{h_{1} h_{2}}= & \frac{\partial f}{\partial y}\left(x, y_{u}, u\right) z_{h_{1} h_{2}}+\frac{\partial^{2} f}{\partial y^{2}}\left(x, y_{u}, u\right) z_{h_{1}} z_{h_{2}} \\
& +\frac{\partial^{2} f}{\partial u \partial y}\left(x, y_{u}, u\right)\left(z_{h_{1}} h_{2}+z_{h_{2}} h_{1}\right)+\frac{\partial^{2} f}{\partial u^{2}}\left(x, y_{u}, u\right) h_{1} h_{2} \quad \text { in } \Omega \\
\partial_{n_{A}} z_{h_{1} h_{2}}= & 0 \text { on } \Gamma
\end{aligned}\right.
$$

Proof. The proof of the existence, uniqueness, and estimate of the solution of (2.1) is standard. Let us prove the differentiability. For that let us start with a homogeneous boundary condition, $g=0$. We consider the space

$$
V(A)=\left\{y \in W^{1, p}(\Omega): A y \in L^{N p /(N+p)}(\Omega), \partial_{n_{A}} y=0\right\}
$$

endowed with the norm

$$
\|y\|_{V(A)}=\|y\|_{W^{1, p}(\Omega)}+\|A y\|_{L^{N p /(N+p)}(\Omega)} .
$$

Let us now define the function

$$
F: V(A) \times L^{\infty}(\Omega) \rightarrow L^{N p /(N+p)}(\Omega), \quad F(y, u)=A y-f(\cdot, y, u) .
$$

Thanks to assumption (A1), $F$ is of class $C^{2}$. Moreover, from Lemma 2.4 it follows that

$$
\frac{\partial F}{\partial y}(y, u)=A-\frac{\partial f}{\partial y}(\cdot, y, u)
$$

is an isomorphism from $V(A)$ to $L^{N p /(N+p)}(\Omega)$. Taking into account that $F(x, y, u)=$ 0 if and only if $y=G(u)$, we can apply the implicit function theorem (see, for instance, [3]) to deduce that $G$ is of class $C^{2}$ and satisfies $F(G(u), u)=0$. From this identity, (2.4) and (2.5) follow easily.

If $g \neq 0$, then we can write $G(u)=y_{u}^{0}+y_{g}=G_{0}(u)+y_{g}$, with $y_{u}^{0}$ and $y_{g}$ solutions of the problems

$$
\begin{gathered}
\left\{\begin{aligned}
A y_{g}=0 & \text { in } \Omega, \\
\partial_{n_{A}} y_{g}=g & \text { on } \Gamma,
\end{aligned}\right. \\
\left\{\begin{aligned}
A y_{u}^{0}=f^{0}\left(x, y_{u}^{0}, u\right) & \text { in } \Omega, \\
\partial_{n_{A}} y_{u}^{0}=0 & \text { on } \Gamma,
\end{aligned}\right.
\end{gathered}
$$

where $f^{0}(x, y, u)=f\left(x, y+y_{g}(x), u\right)$. From the previous argument we have that $G_{0}$ is of class $C^{2}$ and consequently $G$ is $C^{2}$ too, with $G^{\prime}=G_{0}^{\prime}$ and $G^{\prime \prime}=G_{0}^{\prime \prime}$, which concludes the proof. 
As a consequence of this theorem we will get the differentiability of the functionals $J$ and $G_{j}=F_{j} \circ G$ in the next two theorems.

THEOREM 2.6. Let us suppose that (A1) and (A2) hold. Then the functional $J: L^{\infty}(\Omega) \rightarrow \mathbb{R}$ is of class $C^{2}$. Moreover, for every $u, h, h_{1}, h_{2} \in L^{\infty}(\Omega)$,

$$
J^{\prime}(u) h=\int_{\Omega}\left(\frac{\partial L}{\partial u}\left(x, y_{u}, u\right)+\varphi_{0 u} \frac{\partial f}{\partial u}\left(x, y_{u}, u\right)\right) h d x
$$

and

$$
\begin{aligned}
J^{\prime \prime}(u) h_{1} h_{2}=\int_{\Omega}[ & \frac{\partial^{2} L}{\partial y^{2}}\left(x, y_{u}, u\right) z_{1} z_{2}+\frac{\partial^{2} L}{\partial y \partial u}\left(x, y_{u}, u\right)\left(z_{1} h_{2}+z_{2} h_{1}\right) \\
& +\frac{\partial^{2} L}{\partial u^{2}}\left(x, y_{u}, u\right) h_{1} h_{2}+\varphi_{0 u}\left(\frac{\partial^{2} f}{\partial y^{2}}\left(x, y_{u}, u\right) z_{1} z_{2}\right. \\
& \left.\left.+\frac{\partial^{2} f}{\partial y \partial u}\left(x, y_{u}, u\right)\left(z_{1} h_{2}+z_{2} h_{1}\right)+\frac{\partial^{2} f}{\partial u^{2}}\left(x, y_{u}, u\right) h_{1} h_{2}\right)\right] d x
\end{aligned}
$$

where $y_{u}=G(u), \varphi_{0 u} \in W^{1, p}(\Omega)$ is the unique solution of the problem

$$
\left\{\begin{aligned}
A^{*} \varphi & =\frac{\partial f}{\partial y}\left(x, y_{u}, u\right) \varphi+\frac{\partial L}{\partial y}\left(x, y_{u}, u\right) & & \text { in } \Omega, \\
\partial_{n_{A^{*}}} \varphi & =0 & & \text { on } \Gamma,
\end{aligned}\right.
$$

where $A^{*}$ is the adjoint operator of $A$ and $z_{i}=G^{\prime}(u) h_{i}, i=1,2$.

Proof. Let us consider the function $F_{0}: C(\bar{\Omega}) \times L^{\infty}(\Omega) \rightarrow \mathbb{R}$ defined by

$$
F_{0}(y, u)=\int_{\Omega} L(x, y(x), u(x)) d x .
$$

Due to the assumptions on $L$ it is straightforward to prove that $F_{0}$ is of class $C^{2}$. Now, applying the chain rule to $J(u)=F_{0}(G(u), u)$ and using Theorem 2.5 and the fact that $W^{1, p}(\Omega) \subset C(\bar{\Omega})$ for every $p>N$, we get that $J$ is of class $C^{2}$ and

$$
J^{\prime}(u) h=\int_{\Omega}\left(\frac{\partial L}{\partial y}\left(x, y_{u}, u\right) z_{h}+\frac{\partial L}{\partial u}\left(x, y_{u}, u\right) h\right) d x .
$$

Taking $\varphi_{0 u}$ as the solution of (2.8), we deduce (2.6) from previous identity and (2.4). Let us remark that the assumptions on $f$ and $L$ imply the regularity of $\varphi_{0 u}$. The second derivative can be deduced in a similar way, making use of Theorem 2.5 once more.

TheOrem 2.7. Let us suppose that (A1) and (A3) hold. Then for each $j$, the functional $G_{j}=F_{j} \circ G: L^{\infty}(\Omega) \rightarrow \mathbb{R}$ is of class $C^{2}$. Moreover, for every $u, h, h_{1}, h_{2} \in L^{\infty}(\Omega)$,

$$
G_{j}^{\prime}(u) h=\int_{\Omega} \varphi_{j u} \frac{\partial f}{\partial u}\left(x, y_{u}, u\right) h d x
$$

and

$$
G_{j}^{\prime \prime}(u) h_{1} h_{2}=F_{j}^{\prime \prime}\left(y_{u}\right) z_{1} z_{2}
$$

$+\int_{\Omega} \varphi_{j u}\left(\frac{\partial^{2} f}{\partial y^{2}}\left(x, y_{u}, u\right) z_{1} z_{2}+\frac{\partial^{2} f}{\partial y \partial u}\left(x, y_{u}, u\right)\left(z_{1} h_{2}+z_{2} h_{1}\right)+\frac{\partial^{2} f}{\partial u^{2}}\left(x, y_{u}, u\right) h_{1} h_{2}\right) d x$, 
where $y_{u}=G(u), \varphi_{j u} \in W^{1, s^{\prime}}(\Omega)$ is the unique solution of the problem

$$
\left\{\begin{aligned}
A^{*} \varphi_{j u} & =\frac{\partial f}{\partial y}\left(x, y_{u}, u\right) \varphi_{j u}+F_{j}^{\prime}\left(y_{u}\right) & & \text { in } \Omega, \\
\partial_{n_{A^{*}}} \varphi_{j u} & =0 & & \text { on } \Gamma,
\end{aligned}\right.
$$

and $z_{i}=G^{\prime}(u) h_{i}, i=1,2$.

The proof of this theorem is very similar to that of Theorem 2.6. Nevertheless we have to make a comment about (2.11). From assumption (A3) we have that $F^{\prime}(\bar{y}) \in$ $\left(W^{1, s}(\Omega)\right)^{\prime}$; then the boundary problem $(2.11)$ has a unique solution in $W^{1, s^{\prime}}(\Omega)$ in the variational sense, analogous to that of (2.2); see Lemma 2.4. Finally recall that $s<N /(N-1)$; then $s^{\prime}>N$ and therefore $\varphi_{j u} \in W^{1, s^{\prime}}(\Omega) \subset C(\bar{\Omega})$.

3. First and second order optimality conditions in the Lagrangian form. Let us start this section by reformulating problem $(\mathbf{P})$ as follows:

$$
(\mathbf{P})\left\{\begin{array}{l}
\text { Minimize } J(u) \\
u_{a}(x) \leq u(x) \leq u_{b}(x) \text { for a.e. } x \in \Omega, \\
G_{j}(u)=0,1 \leq j \leq n_{e} \\
G_{j}(u) \leq 0, n_{e}+1 \leq j \leq n_{e}+n_{i}
\end{array}\right.
$$

where we are using the functions introduced in the previous section $G_{j}=F_{j} \circ G$. We now apply the results obtained in [7]. In order to deduce the first and second order optimality conditions of an optimization problem, it is necessary to make a regularity assumption. This is our first goal. Given $\varepsilon>0$, we denote the set of $\varepsilon$-inactive constraints by

$$
\Omega_{\varepsilon}=\left\{x \in \Omega: u_{a}(x)+\varepsilon \leq \bar{u}(x) \leq u_{b}(x)-\varepsilon\right\} .
$$

We say that a feasible control $\bar{u}$ is regular if the following assumption is fulfilled:

$$
\left\{\begin{array}{l}
\exists \varepsilon_{\bar{u}}>0 \text { and }\left\{\bar{h}_{j}\right\}_{j \in I_{0}} \subset L^{\infty}(\Omega), \text { with supp } \bar{h}_{j} \subset \Omega_{\varepsilon_{\bar{u}}} \text {, such that } \\
G_{i}^{\prime}(\bar{u}) \bar{h}_{j}=\delta_{i j}, \quad i, j \in I_{0},
\end{array}\right.
$$

where

$$
I_{0}=\left\{j \leq m \mid G_{j}(\bar{u})=0\right\} .
$$

$I_{0}$ is the set of indices corresponding to active constraints. Associated to (P) we define the Lagrangian function

$$
\mathcal{L}(u, \lambda)=J(u)+\sum_{j=1}^{n_{e}+n_{i}} \lambda_{j} G_{j}(u)
$$

Obviously (3.1) is equivalent to the independence of the derivatives $\left\{G_{j}^{\prime}(\bar{u})\right\}_{j \in I_{0}}$ in $L^{1}\left(\Omega_{\varepsilon_{\bar{u}}}\right)$. Under this assumption we can derive the first order necessary conditions for optimality in a qualified form. For the proof the reader is referred to Bonnans and Casas [1] or Clarke [11]; see also Mateos [17].

THEOREM 3.1. Let us assume that $\bar{u}$ is a local solution of $(\mathbf{P})$ and (3.1) holds. Then there exist real numbers $\left\{\bar{\lambda}_{j}\right\}_{j=1}^{n_{e}+n_{i}}$ such that

$$
\bar{\lambda}_{j} \geq 0, \quad n_{e}+1 \leq j \leq n_{e}+n_{i}, \bar{\lambda}_{j}=0 \text { if } G_{j}(\bar{u})<0 ;
$$




$$
\frac{\partial \mathcal{L}}{\partial u}(\bar{u}, \bar{\lambda})(u-\bar{u}) \geq 0 \quad \text { for all } u_{a} \leq u \leq u_{b} .
$$

Denoting by $\bar{\varphi}_{0}$ and $\bar{\varphi}_{j}$ the solutions of (2.8) and (2.11) corresponding to $\bar{u}$ and setting

$$
\bar{\varphi}=\bar{\varphi}_{0}+\sum_{j=1}^{n_{e}+n_{i}} \bar{\lambda}_{j} \bar{\varphi}_{j}
$$

we deduce from Theorems 2.6 and 2.7 and the definition of $\mathcal{L}$ that

$$
\begin{aligned}
\frac{\partial \mathcal{L}}{\partial u}(\bar{u}, \bar{\lambda}) h & =\int_{\Omega}\left(\frac{\partial L}{\partial u}(x, \bar{y}, \bar{u})+\bar{\varphi}_{0} \frac{\partial f}{\partial u}(x, \bar{y}, \bar{u})\right) h d x+\sum_{j=1}^{n_{e}+n_{i}} \bar{\lambda}_{j} \int_{\Omega} \bar{\varphi}_{j} \frac{\partial f}{\partial u}(x, \bar{y}, \bar{u}) h d x \\
& =\int_{\Omega}\left(\frac{\partial L}{\partial u}(x, \bar{y}, \bar{u})+\bar{\varphi} \frac{\partial f}{\partial u}(x, \bar{y}, \bar{u})\right) h d x \\
(3.5) \quad & =\int_{\Omega} d(x) h(x) d x \quad \forall h \in L^{\infty}(\Omega),
\end{aligned}
$$

where $\bar{y}=G(\bar{u})=y_{\bar{u}}$ and

$$
d(x)=\frac{\partial L}{\partial u}(x, \bar{y}(x), \bar{u}(x))+\bar{\varphi}(x) \frac{\partial f}{\partial u}(x, \bar{y}(x), \bar{u}(x))=\frac{\partial H}{\partial u}(x, \bar{y}(x), \bar{u}(x), \bar{\varphi}(x)),
$$

$H: \Omega \times \mathbb{R}^{3} \longrightarrow \mathbb{R}$ being the Hamiltonian associated to the control problem $(\mathbf{P})$,

$$
H(x, y, u, \varphi)=L(x, y, u)+\varphi f(x, y, u) .
$$

From (3.3) we deduce that

$$
d(x)=\left\{\begin{array}{cl}
0 & \text { for a.e. } x \in \Omega, \text { where } u_{a}(x)<\bar{u}(x)<u_{b}(x), \\
\geq 0 & \text { for a.e. } x \in \Omega, \text { where } \bar{u}(x)=u_{a}(x) \\
\leq 0 & \text { for a.e. } x \in \Omega, \text { where } \bar{u}(x)=u_{b}(x)
\end{array}\right.
$$

Remark 3.2. From (3.3), (3.7), and assumption (3.1) we get

$$
\int_{\Omega}\left(\frac{\partial L}{\partial u}(x, \bar{y}(x), \bar{u}(x))+\bar{\varphi}_{0}(x) \frac{\partial f}{\partial u}(x, \bar{y}(x), \bar{u}(x))\right) \bar{h}_{j}(x) d x+\bar{\lambda}_{j}=\frac{\partial \mathcal{L}}{\partial u}(\bar{u}, \bar{\lambda}) \bar{h}_{j}=0,
$$

which implies the uniqueness of the Lagrange multipliers provided in Theorem 3.1.

Associated with $d$ we set

$$
\Omega^{0}=\{x \in \Omega:|d(x)|>0\} .
$$

Given $\left\{\bar{\lambda}_{j}\right\}_{j=1}^{n_{e}+n_{i}}$ by Theorem 3.1 we define the cone of critical directions

$$
C_{\bar{u}}^{0}=\left\{h \in L^{\infty}(\Omega) \text { satisfying (3.10) and } h(x)=0 \text { for a.e. } x \in \Omega^{0}\right\} \text {, }
$$

with

$$
\left\{\begin{array}{l}
G_{j}^{\prime}(\bar{u}) h=0 \text { if }\left(j \leq n_{e}\right) \text { or }\left(j>n_{e}, G_{j}(\bar{u})=0, \text { and } \bar{\lambda}_{j}>0\right) \\
G_{j}^{\prime}(\bar{u}) h \leq 0 \text { if } j>n_{e}, G_{j}(\bar{u})=0, \text { and } \bar{\lambda}_{j}=0 \\
h(x)= \begin{cases}\geq 0 & \text { if } \bar{u}(x)=u_{a}(x) \\
\leq 0 & \text { if } \bar{u}(x)=u_{b}(x)\end{cases}
\end{array}\right.
$$


Now we are ready to state the second order necessary optimality conditions.

THEOREM 3.3. Let us assume that $\bar{u}$ is a local solution of $(\mathbf{P}),(3.1)$ holds, and $\left\{\bar{\lambda}_{j}\right\}_{j=1}^{n_{e}+n_{i}}$ are the Lagrange multipliers satisfying (3.2) and (3.3). Then the following inequality is satisfied:

$$
\frac{\partial^{2} \mathcal{L}}{\partial u^{2}}(\bar{u}, \bar{\lambda}) h^{2} \geq 0 \quad \forall h \in C_{\bar{u}}^{0}
$$

This theorem follows from Theorem 2.2 of [7]. Indeed it is enough to check the assumptions (A1) and (A2) of such a paper. (A1) says that $J^{\prime}(\bar{u})$ and $G_{j}^{\prime}(\bar{u})$ must be continuous functionals on $L^{2}(\Omega)$, which is an immediate consequence of Theorems 2.6 and 2.7. Assumption (A2) of [7] says that

$$
\frac{\partial^{2} \mathcal{L}}{\partial u^{2}}(\bar{u}, \bar{\lambda}) h_{k}^{2} \longrightarrow \frac{\partial^{2} \mathcal{L}}{\partial u^{2}}(\bar{u}, \bar{\lambda}) h^{2}
$$

whenever $\left\{h_{k}\right\}_{k=1}^{\infty}$ is bounded in $L^{\infty}(\Omega)$ and $h_{k}(x) \rightarrow h(x)$ a.e. in $\Omega$. Taking into account that

$$
\begin{aligned}
\frac{\partial^{2} \mathcal{L}}{\partial u^{2}}(\bar{u}, \bar{\lambda}) h^{2}= & \sum_{j=1}^{n_{e}+n_{i}} \bar{\lambda}_{j} F_{j}^{\prime \prime}(\bar{y}) z_{h}^{2} \\
& +\int_{\Omega}\left(\frac{\partial^{2} L}{\partial y^{2}}(x, \bar{y}, \bar{u})+\bar{\varphi} \frac{\partial^{2} f}{\partial y^{2}}(x, \bar{y}, \bar{u})\right) z_{h}^{2} d x \\
& +\int_{\Omega}\left(\frac{\partial^{2} L}{\partial y \partial u}(x, \bar{y}, \bar{u})+\bar{\varphi} \frac{\partial^{2} f}{\partial y \partial u}(x, \bar{y}, \bar{u})\right) z_{h} h d x \\
& +\int_{\Omega}\left(\frac{\partial^{2} L}{\partial u^{2}}(x, \bar{y}, \bar{u})+\bar{\varphi} \frac{\partial^{2} f}{\partial u^{2}}(x, \bar{y}, \bar{u})\right) h^{2} d x
\end{aligned}
$$

where $z_{h}$ is the solution of (2.4) corresponding to the pair $(\bar{y}, \bar{u})$, the desired convergence property follows from the boundedness of the second derivatives of $L$ and $f$ along with the convergence $z_{h_{k}} \rightarrow z_{h}$ in $W^{1, q}(\Omega) \subset L^{2}(\Omega)$ and our assumption (A3).

In order to obtain the sufficient second order optimality conditions for problem $(\mathbf{P})$, we need to check some additional properties of the first and second derivatives of $J$ and $G_{j}$. Let us take a ball in $L^{\infty}(\Omega), B_{\rho}(\bar{u})$. From Theorem 2.5, we deduce the existence of a constant $C_{\rho}>0$ such that $\left\{y_{u}\right\}_{u \in B_{\rho}(\bar{u})}$ is uniformly bounded by $C_{\rho}$ in the $W^{1, p}(\Omega)$ norm and therefore in the $L^{\infty}(\Omega)$ norm too. This implies the uniform boundedness of the derivatives of $f$ at every point $\left(y_{u}, u\right)$, for $u \in B_{\rho}(\bar{u})$, as well as the boundedness of the second derivatives of $L$ and the domination of the first derivatives by some functions $\psi_{\rho} \in L^{N p /(N+p)}(\Omega)$ and $\psi_{\rho}^{*} \in L^{2}(\Omega)$. Then from Lemma 2.4 we deduce that $\left\{\varphi_{j u}\right\}_{u \in B_{\rho}(\bar{u})}$ are bounded in $W^{1, p}(\Omega) \subset L^{\infty}(\Omega)$ for $j=0$ and $W^{1, s^{\prime}}(\Omega) \subset L^{\infty}(\Omega)$ for $1 \leq j \leq n_{e}+n_{i}$, respectively. Finally, using Lemma 2.4 once more, we get that

$$
\left\|z_{h}\right\|_{W^{1, q}(\Omega)} \leq C\|h\|_{L^{2}(\Omega)}
$$

which follows from the imbedding $L^{2}(\Omega) \subset\left(W^{1, q^{\prime}}(\Omega)\right)^{\prime}$ due to the fact $q<2 N /(N-2)$. Collecting all these things, we get the existence of constants $M_{j, 1}, M_{j, 2}>0$, with 
$0 \leq j \leq n_{e}+n_{i}$, such that for every $u \in B_{\rho}(\bar{u})$ and all $h, h_{1}, h_{2} \in L^{\infty}(\Omega)$ we have

$$
\left\{\begin{array}{l}
\left|J^{\prime}(u) h\right| \leq M_{0,1}\|h\|_{L^{2}(\Omega)}, \quad\left|G_{j}^{\prime}(u) h\right| \leq M_{j, 1}\|h\|_{L^{2}(\Omega)}, \\
\left|J^{\prime \prime}(u) h_{1} h_{2}\right| \leq M_{0,2}\left\|h_{1}\right\|_{L^{2}(\Omega)}\left\|h_{2}\right\|_{L^{2}(\Omega)} \\
\left|G_{j}^{\prime \prime}(u) h_{1} h_{2}\right| \leq M_{j, 2}\left\|h_{1}\right\|_{L^{2}(\Omega)}\left\|h_{2}\right\|_{L^{2}(\Omega)}
\end{array}\right.
$$

We have to check a last condition, which is established in the following lemma.

LEMma 3.4. For every $\delta>0$ there exists $\varepsilon \in(0, \rho)$ such that for every $h \in L^{\infty}(\Omega)$ and $\|u-\bar{u}\|_{\infty}<\varepsilon$ the following inequality is fulfilled:

$$
\left|\left[\frac{\partial^{2} \mathcal{L}}{\partial u^{2}}(u, \bar{\lambda})-\frac{\partial^{2} \mathcal{L}}{\partial u^{2}}(\bar{u}, \bar{\lambda})\right] h^{2}\right| \leq \delta\|h\|_{L^{2}(\Omega)}^{2} .
$$

Proof. Let us take $h \in L^{\infty}(\Omega)$ and $\delta>0$. We are going to check that

$$
\begin{aligned}
& \quad\left|\left[\frac{\partial^{2} \mathcal{L}}{\partial u^{2}}(v, \bar{\lambda})-\frac{\partial^{2} \mathcal{L}}{\partial u^{2}}(\bar{u}, \bar{\lambda})\right] h^{2}\right| \\
& \leq \int_{\Omega}\left|\frac{\partial^{2} L}{\partial u^{2}}\left(x, y_{v}, v\right)+\varphi_{v} \frac{\partial^{2} f}{\partial u^{2}}\left(x, y_{v}, v\right)-\frac{\partial^{2} L}{\partial u^{2}}(x, \bar{y}, \bar{u})-\bar{\varphi} \frac{\partial^{2} f}{\partial u^{2}}(x, \bar{y}, \bar{u})\right| h^{2} d x \\
& \quad+\int_{\Omega} \mid\left(\frac{\partial^{2} L}{\partial y \partial u}\left(x, y_{v}, v\right)+\varphi_{v} \frac{\partial^{2} f}{\partial y \partial u}\left(x, y_{v}, v\right)\right) z_{h} \\
& \quad-\left(\frac{\partial^{2} L}{\partial y \partial u}(x, \bar{y}, \bar{u})+\bar{\varphi} \frac{\partial^{2} f}{\partial y \partial u}(x, \bar{y}, \bar{u})\right) \bar{z}_{h}|| h \mid d x \\
& \quad+\int_{\Omega} \mid\left(\frac{\partial^{2} L}{\partial y^{2}}\left(x, y_{v}, v\right)+\varphi_{v} \frac{\partial^{2} f}{\partial y^{2}}\left(x, y_{v}, v\right)\right) z_{h}^{2} \\
& \quad-\left(\frac{\partial^{2} L}{\partial y^{2}}(x, \bar{y}, \bar{u})+\bar{\varphi} \frac{\partial^{2} f}{\partial y^{2}}(x, \bar{y}, \bar{u})\right) \bar{z}_{h}^{2} \mid d x \\
& \quad+\sum_{j=1}^{n_{i}+n i}\left|\bar{\lambda}_{j}\right|\left|F_{j}^{\prime \prime}\left(y_{v}\right) z_{h}^{2}-F_{j}^{\prime \prime}(\bar{y}) \bar{z}_{h}^{2}\right| \leq \delta\|h\|_{L^{2}(\Omega)}^{2},
\end{aligned}
$$

supposing $\|v-\bar{u}\|_{L^{\infty}(\Omega)}<\varepsilon$ with $\varepsilon$ small enough, where

$$
\left\{\begin{aligned}
A z_{h} & =\frac{\partial f}{\partial y}\left(x, y_{v}, v\right) z_{h}+\frac{\partial f}{\partial u}\left(x, y_{v}, v\right) h & & \text { in } \Omega, \\
\partial_{n_{A}} z_{h} & =0 & & \text { on } \Gamma,
\end{aligned}\right.
$$

and

$$
\left\{\begin{aligned}
A \bar{z}_{h} & =\frac{\partial f}{\partial y}(x, \bar{y}, \bar{u}) \bar{z}_{h}+\frac{\partial f}{\partial u}(x, \bar{y}, \bar{u}) h & & \text { in } \Omega, \\
\partial_{n_{A}} \bar{z}_{h} & =0 & & \text { on } \Gamma .
\end{aligned}\right.
$$

We discuss every term in a separate way. The inequality

$$
\left\|\frac{\partial^{2} L}{\partial u^{2}}\left(x, y_{v}, v\right)+\varphi_{v} \frac{\partial^{2} f}{\partial u^{2}}\left(x, y_{v}, v\right)-\frac{\partial^{2} L}{\partial u^{2}}(x, \bar{y}, \bar{u})-\bar{\varphi} \frac{\partial^{2} f}{\partial u^{2}}(x, \bar{y}, \bar{u})\right\|_{L^{\infty}(\Omega)}<\frac{\delta}{4}
$$


is a direct consequence of the continuity $v \in L^{\infty}(\Omega) \longrightarrow \varphi_{v} \in W^{1, \min \left\{s^{\prime}, p\right\}}(\Omega) \subset C(\bar{\Omega})$ (see Lemma 2.4 and Theorems 2.6 and 2.7) and the continuity properties of the second derivatives of $f$ and $L$ assumed in (A1) and (A2), as well as assumption (A3).

Let us study the second term of (3.15). Hölder's inequality leads us to

$$
\begin{gathered}
\int_{\Omega} \mid\left(\frac{\partial^{2} L}{\partial y \partial u}\left(x, y_{v}, v\right)+\varphi_{v} \frac{\partial^{2} f}{\partial y \partial u}\left(x, y_{v}, v\right)\right) z_{h} \\
-\left(\frac{\partial^{2} L}{\partial y \partial u}(x, \bar{y}, \bar{u})+\bar{\varphi} \frac{\partial^{2} f}{\partial y \partial u}(x, \bar{y}, \bar{u})\right) \bar{z}_{h}|| h \mid d x \\
\leq\|h\|_{L^{2}(\Omega)}\left(\left\|\frac{\partial^{2} L}{\partial y \partial u}\left(x, y_{v}, v\right)-\frac{\partial^{2} L}{\partial y \partial u}(x, \bar{y}, \bar{u})\right\|_{L^{\infty}(\Omega)}\left\|z_{h}\right\|_{L^{2}(\Omega)}\right. \\
+\left\|\frac{\partial^{2} L}{\partial y \partial u}(x, \bar{y}, \bar{u})\right\|_{L^{\infty}(\Omega)}\left\|z_{h}-\bar{z}_{h}\right\|_{L^{2}(\Omega)} \\
+\left\|\varphi_{v} \frac{\partial^{2} f}{\partial y \partial u}\left(x, y_{v}, v\right)-\bar{\varphi} \frac{\partial^{2} f}{\partial y \partial u}(x, \bar{y}, \bar{u})\right\|_{L^{\infty}(\Omega)}\left\|z_{h}\right\|_{L^{2}(\Omega)} \\
\left.+\left\|\bar{\varphi} \frac{\partial^{2} f}{\partial y \partial u}(x, \bar{y}, \bar{u})\right\|_{L^{\infty}(\Omega)}\left\|z_{h}-\bar{z}_{h}\right\|_{L^{2}(\Omega)}\right)<\frac{\delta}{4}\|h\|_{L^{2}(\Omega)}^{2},
\end{gathered}
$$

the last inequality being a consequence of (A1) and (A2) along with the estimates

$$
\left\|z_{h}\right\|_{L^{2}(\Omega)}+\left\|\bar{z}_{h}\right\|_{L^{2}(\Omega)} \leq C_{1}\left(\left\|z_{h}\right\|_{W^{1, q}(\Omega)}+\left\|\bar{z}_{h}\right\|_{W^{1, q}(\Omega)}\right) \leq C_{2}\|h\|_{L^{2}(\Omega)}
$$

and

$$
\left\|z_{h}-\bar{z}_{h}\right\|_{L^{2}(\Omega)} \leq C_{1}\left\|z_{h}-\bar{z}_{h}\right\|_{W^{1, q}(\Omega)} \leq O(\varepsilon)\|h\|_{L^{2}(\Omega)},
$$

with $O(\varepsilon) \rightarrow 0$ when $\varepsilon \rightarrow 0$. Let us notice that (3.16) follows from the inequalities $2 N /(N+2) \leq q<2 N /(N-2)$, Sobolev imbeddings, and Lemma 2.4.

Analogously we have

$$
\begin{aligned}
& \int_{\Omega}\left|\left(\frac{\partial^{2} L}{\partial y^{2}}\left(x, y_{v}, v\right)+\varphi_{v} \frac{\partial^{2} f}{\partial y^{2}}\left(x, y_{v}, v\right)\right) z_{h}^{2}-\left(\frac{\partial^{2} L}{\partial y^{2}}(x, \bar{y}, \bar{u})+\bar{\varphi} \frac{\partial^{2} f}{\partial y^{2}}(x, \bar{y}, \bar{u})\right) \bar{z}_{h}^{2}\right| d x \\
\leq & \left\|\frac{\partial^{2} L}{\partial y^{2}}\left(x, y_{v}, v\right)-\frac{\partial^{2} L}{\partial y^{2}}(x, \bar{y}, \bar{u})\right\|_{L^{\infty}(\Omega)}\left\|z_{h}\right\|_{L^{2}(\Omega)}^{2} \\
& +\left\|\frac{\partial^{2} L}{\partial y^{2}}(x, \bar{y}, \bar{u})\right\|_{L^{\infty}(\Omega)}\left\|z_{h}-\bar{z}_{h}\right\|_{L^{2}(\Omega)}\left\|z_{h}+\bar{z}_{h}\right\|_{L^{2}(\Omega)} \\
& +\left\|\varphi_{v} \frac{\partial^{2} f}{\partial y^{2}}\left(x, y_{v}, v\right)-\bar{\varphi} \frac{\partial^{2} f}{\partial y^{2}}(x, \bar{y}, \bar{u})\right\|_{L^{\infty}(\Omega)}\left\|z_{h}\right\|_{L^{2}(\Omega)}^{2} \\
& +\left\|\bar{\varphi} \frac{\partial^{2} f}{\partial y^{2}}(x, \bar{y}, \bar{u})\right\|_{L^{\infty}(\Omega)}\left\|z_{h}-\bar{z}_{h}\right\|_{L^{2}(\Omega)}\left\|z_{h}+\bar{z}_{h}\right\|_{L^{2}(\Omega)}<\frac{\delta}{4}\|h\|_{L^{2}(\Omega)}^{2},
\end{aligned}
$$

thanks again to (A1), (A2), (3.16), and (3.17).

For the fourth term of (3.15) it is enough to take into account assumption (A3), and once more (3.16) and (3.17), and to use the inequality

$$
\begin{aligned}
& \left|F_{j}^{\prime \prime}\left(y_{v}\right) z_{h}^{2}-F_{j}^{\prime \prime}(\bar{y}) \bar{z}_{h}^{2}\right|=\left|F_{j}^{\prime \prime}\left(y_{v}\right)\left(z_{h}^{2}-\bar{z}_{h}^{2}\right)+\left(F_{j}^{\prime \prime}\left(y_{v}\right)-F_{j}^{\prime \prime}(\bar{y})\right) \bar{z}_{h}^{2}\right| \\
& \quad \leq\left|F_{j}^{\prime \prime}\left(y_{v}\right)\left(z_{h}+\bar{z}_{h}\right)\left(z_{h}-\bar{z}_{h}\right)\right|+\left|\left(F_{j}^{\prime \prime}\left(y_{v}\right)-F_{j}^{\prime \prime}(\bar{y})\right) \bar{z}_{h}^{2}\right| .
\end{aligned}
$$


Before writing the sufficient optimality conditions, we have to fix some notation. Analogously to (3.8) and (3.9), we define for every $\tau>0$

$$
\Omega^{\tau}=\{x \in \Omega:|d(x)|>\tau\}
$$

and

$$
C_{\bar{u}}^{\tau}=\left\{h \in L^{\infty}(\Omega) \text { satisfying }(3.10) \text { and } h(x)=0 \text { for a.e. } x \in \Omega^{\tau}\right\} .
$$

The next theorem provides the second order sufficient optimality conditions of problem $(\mathbf{P})$.

TheOREM 3.5. Let $\bar{u}$ be a feasible point for problem (P) satisfying (3.2) and (3.3) and let us suppose that assumption (3.1) holds. Let us also assume that

$$
\frac{\partial^{2} \mathcal{L}}{\partial u^{2}}(\bar{u}, \bar{\lambda}) h^{2} \geq \delta\|h\|_{L^{2}(\Omega)}^{2} \quad \forall h \in C_{\bar{u}}^{\tau}
$$

for some $\delta>0$ and $\tau>0$ given. Then there exist $\varepsilon>0$ and $\alpha>0$ such that $J(\bar{u})+\alpha\|u-\bar{u}\|_{L^{2}(\Omega)}^{2} \leq J(u)$ for every feasible point $u$ for $(\mathbf{P})$, with $\|u-\bar{u}\|_{L^{\infty}(\Omega)}<\varepsilon$.

Relations (3.13) and (3.14) prove that the hypotheses of Corollary 3.3 of [7] are fulfilled, which leads straightforwardly to the above theorem. In that paper it is also proved that we can not relax the sufficient condition by taking $\tau=0$; see also Dunn [12].

The last two theorems concerning the necessary and sufficient second order optimality conditions involve two norms: those of $L^{2}(\Omega)$ and $L^{\infty}(\Omega)$. This is motivated by the so-called two norms discrepancy; see, for instance; A. Ioffe [15] and H. Maurer [18]. In particular, the cones $C_{\bar{u}}^{\tau}$, for $\tau \geq 0$, as defined in (3.8) and (3.19), are subsets of $L^{\infty}(\Omega)$, but only the $L^{2}(\Omega)$-norm of the elements of $C_{\bar{u}}^{\tau}$ is involved in the optimality conditions (3.11) and (3.20). Now there is a natural question. Let us define for each $\tau \geq 0$

$$
C_{\bar{u}, L^{2}(\Omega)}^{\tau}=\left\{h \in L^{2}(\Omega) \text { satisfying }(3.10) \text { and } h(x)=0 \text { for a.e. } x \in \Omega^{\tau}\right\} .
$$

Can we replace $C_{\bar{u}}^{\tau}$ by $C_{\bar{u}, L^{2}(\Omega)}^{\tau}$ in Theorems 3.3 and 3.5 ? The next proposition provides a positive answer.

Proposition 3.6. Let us assume that (3.1) holds. Then $C_{\bar{u}, L^{2}(\Omega)}^{\tau}=\bar{C}_{\bar{u}}^{\tau}$, where $\bar{C}_{\bar{u}}^{\tau}$ denotes the closure of $C_{\bar{u}}^{\tau}$ in $L^{2}(\Omega)$.

Proof. Since $C_{\bar{u}, L^{2}(\Omega)}^{\tau}$ is closed in $L^{2}(\Omega)$, we obviously have $\bar{C}_{\bar{u}}^{\tau} \subset C_{\bar{u}, L^{2}(\Omega)}^{\tau}$. Let us prove the reverse inclusion. Let $h \in C_{\bar{u}, L^{2}(\Omega)}^{\tau}$. We are going to obtain a sequence $\left\{h_{k}\right\}_{k=1}^{\infty} \subset C_{\bar{u}}^{\tau}$ such that $h_{k} \rightarrow h$ in $L^{2}(\Omega)$. Let us take

$$
\hat{h}_{k}=\left\{\begin{array}{ccc}
+k & \text { if } & h(x)>+k, \\
h(x) & \text { if } & |h(x)| \leq k, \\
-k & \text { if } & h(x)<-k
\end{array}\right.
$$

For every $j \in I_{0}$ let us set

$$
\alpha_{k j}=G_{j}^{\prime}(\bar{u}) \hat{h}_{k}-G_{j}^{\prime}(\bar{u}) h .
$$

It is clear that $\hat{h}_{k} \rightarrow h$ in $L^{2}(\Omega)$ and $\alpha_{k j} \rightarrow 0$ for every $j \in I_{0}$. Finally we define

$$
h_{k}=\hat{h}_{k}-\sum_{j \in I_{0}} \alpha_{k j} \bar{h}_{j}
$$


where $\bar{h}_{j}$ is given in (3.1). It is obvious that $h_{k} \rightarrow h$ in $L^{2}(\Omega)$ and $\left\{h_{k}\right\}_{k=1}^{\infty} \subset L^{\infty}(\Omega)$. Let us prove that $h_{k} \in C_{\bar{u}}^{\tau}$ for every $k$. First of all, $h_{k}(x)=0$ for almost every $x \in \Omega^{\tau}$. Indeed, since $h \in C_{\bar{u}, L^{2}(\Omega)}^{\tau}$, then $h(x)=0$ for almost every $x \in \Omega^{\tau}$; consequently $\hat{h}_{k}$ keeps the same property. On the other hand, the support of $\bar{h}_{j}$ is in $\Omega_{\varepsilon_{\bar{u}}}$, and $d(x)=0$ for almost every $x \in \Omega_{\varepsilon_{\bar{u}}}$; therefore $\bar{h}_{j}(x)=0$ for almost all $x \in \Omega^{\tau}$. Hence $h_{k}(x)$ also vanishes almost everywhere in $\Omega^{\tau}$. Moreover, since $h$ and $\hat{h}_{k}$ have the same sign, it follows that $h_{k}(x)=\hat{h}_{k}(x) \geq 0$ if $\bar{u}(x)=u_{a}(x)$. Analogously, if $\bar{u}(x)=u_{b}(x)$, then $h_{k}(x)=\hat{h}_{k}(x) \leq 0$. Finally, let us fix $j \in I_{0}$

$$
G_{j}^{\prime}(\bar{u}) h_{k}=G_{j}^{\prime}(\bar{u}) \hat{h}_{k}-\sum_{i \in I_{0}} \alpha_{k i} G_{j}^{\prime}(\bar{u}) \bar{h}_{i}=G_{j}^{\prime}(\bar{u}) \hat{h}_{k}-\alpha_{k j}=G_{j}^{\prime}(\bar{u}) h .
$$

Using the fact that $h$ is in the cone of critical directions of $L^{2}(\Omega)$, we deduce that $h_{k}$ satisfies in the same way as $h$ the conditions on the derivatives of $G_{j}$, for every $j$, which proves that $h_{k} \in C_{\bar{u}}^{\tau}$.

Remark 3.7. As a consequence of the previous proposition and the fact that $\frac{\partial^{2} \mathcal{L}}{\partial u^{2}}(\bar{u}, \bar{\lambda})$ is a bilinear and continuous form in $L^{2}(\Omega)$, we get the following equivalences:

$$
\frac{\partial^{2} \mathcal{L}}{\partial u^{2}}(\bar{u}, \bar{\lambda}) h^{2} \geq 0 \quad \forall h \in C_{\bar{u}}^{0} \Longleftrightarrow \frac{\partial^{2} \mathcal{L}}{\partial u^{2}}(\bar{u}, \bar{\lambda}) h^{2} \geq 0 \quad \forall h \in C_{\bar{u}, L^{2}(\Omega)}^{0}
$$

and

$$
\frac{\partial^{2} \mathcal{L}}{\partial u^{2}}(\bar{u}, \bar{\lambda}) h^{2} \geq \delta\|h\|_{L^{2}(\Omega)}^{2} \quad \forall h \in C_{\bar{u}}^{\tau} \Longleftrightarrow \frac{\partial^{2} \mathcal{L}}{\partial u^{2}}(\bar{u}, \bar{\lambda}) h^{2} \geq \delta\|h\|_{L^{2}(\Omega)}^{2} \quad \forall h \in C_{\bar{u}, L^{2}(\Omega)}^{\tau} .
$$

4. First and second order optimality conditions involving the Hamiltonian. As in the previous section, we denote with $H: \Omega \times \mathbb{R}^{3} \longrightarrow \mathbb{R}$ the Hamiltonian associated to the control problem $(\mathbf{P})$ :

$$
H(x, y, u, \varphi)=L(x, y, u)+\varphi f(x, y, u) .
$$

Pontryagin's principle for $(\mathbf{P})$ is formulated in terms of $H$ in the next proposition.

Proposition 4.1. Let $\bar{u}$ be a solution of $(\mathbf{P})$. Suppose that the assumptions (A1)-(A3) and (3.1) hold. Then there exist real numbers $\bar{\lambda}_{j}, j=1, \ldots, n_{i}+n_{e}$, and functions $\bar{y} \in W^{1, p}(\Omega), \bar{\varphi} \in W^{1, \min \left\{s^{\prime}, p\right\}}(\Omega)$ such that

$$
\begin{gathered}
\bar{\lambda}_{j} \geq 0, \quad n_{e}+1 \leq j \leq n_{e}+n_{i}, \quad \bar{\lambda}_{j} F_{j}(\bar{y})=0, \\
\left\{\begin{array}{cl}
A \bar{y}=f(x, \bar{y}(x), \bar{u}(x)) \quad \text { in } \Omega, \\
\partial_{n_{A}} \bar{y}=0 & \text { on } \Gamma,
\end{array}\right. \\
\left\{\begin{aligned}
A^{*} \bar{\varphi}=\frac{\partial f}{\partial y}(x, \bar{y}, \bar{u}) \bar{\varphi}+\frac{\partial L}{\partial y}(x, \bar{y}, \bar{u})+\sum_{j=1}^{n_{e}+n_{i}} \bar{\lambda}_{j} F_{j}^{\prime}(\bar{y}) & \text { in } \Omega, \\
\partial_{n_{A^{*}} \bar{\varphi}}=0 & \text { on } \Gamma,
\end{aligned}\right.
\end{gathered}
$$

and for a.e. $x \in \Omega$

$$
H(x, \bar{y}(x), \bar{u}(x), \bar{\varphi}(x))=\min _{k \in\left[u_{a}(x), u_{b}(x)\right]} H(x, \bar{y}(x), k, \bar{\varphi}(x)) .
$$


Proof. Let us define $H_{\nu}: \Omega \times \mathbb{R}^{3} \longrightarrow \mathbb{R}$ by

$$
H_{\nu}(x, y, u, \varphi)=\nu L(x, y, u)+\varphi f(x, y, u) .
$$

It is known (see Casas [4], Casas, Raymond, and Zidani [6], Li and Yong [16], or Mateos [17]) that there exist $\bar{\nu} \geq 0, \bar{\lambda}=\left(\bar{\lambda}_{j}\right)_{1 \leq j \leq n_{i}+n_{e}}$, and functions $\bar{y} \in W^{1, p}(\Omega)$, $\bar{\varphi} \in W^{1, \min \left\{s^{\prime}, p\right\}}(\Omega)$ such that $(\bar{\nu}, \bar{\lambda}) \neq 0,(4.1)$ and (4.2) hold, and

$$
\left\{\begin{aligned}
A^{*} \bar{\varphi} & =\frac{\partial f}{\partial y}(x, \bar{y}, \bar{u}) \bar{\varphi}+\bar{\nu} \frac{\partial L}{\partial y}(x, \bar{y}, \bar{u})+\sum_{j=1}^{n_{e}+n_{i}} \bar{\lambda}_{j} F_{j}^{\prime}(\bar{y}) & & \text { in } \Omega \\
\partial_{n_{A^{*}} \bar{\varphi}} & =0 & & \text { on } \Gamma
\end{aligned}\right.
$$

and

(4.6) $H_{\bar{\nu}}(x, \bar{y}(x), \bar{u}(x), \bar{\varphi}(x))=\min _{k \in\left[u_{a}(x), u_{b}(x)\right]} H_{\bar{\nu}}(x, \bar{y}(x), k, \bar{\varphi}(x))$ for a.e. $x \in \Omega$.

In the case $\bar{\nu}>0$, we can rename $\bar{\lambda} / \bar{\nu}$ by $\bar{\lambda}$ and obtain (4.1)-(4.5). So it is enough to prove that $\bar{\nu} \neq 0$. Let us argue by contradiction and let us suppose that $\bar{\nu}=0$. Since $H_{\bar{\nu}}$ is $C^{1}$ with respect to $(y, u) \in \mathbb{R} \times \mathbb{R}$, we deduce from (4.6) and Theorem 2.7 that for every $u_{a} \leq u \leq u_{b}$

$$
\sum_{j=1}^{n_{i}+n_{e}} \bar{\lambda}_{j} G_{j}^{\prime}(\bar{u})(u-\bar{u})=\int_{\Omega} \frac{\partial H_{\bar{\nu}}}{\partial u}(x, \bar{y}(x), \bar{u}(x), \bar{\varphi}(x))(u(x)-\bar{u}(x)) d x \geq 0 .
$$

Let us take $\bar{h}_{j}$ as defined in assumption (3.1) and $|\rho|<\varepsilon$ small enough such that $u_{a} \leq u=\bar{u}+\rho \bar{h}_{j} \leq u_{b}$; then

$$
\rho \bar{\lambda}_{j}=\sum_{i=1}^{n_{i}+n_{e}} \bar{\lambda}_{i} G_{i}^{\prime}(\bar{u})(u-\bar{u}) \geq 0 .
$$

By taking $\rho$ positive and negative, respectively, we get that $\bar{\lambda}_{j}=0$ for every $j \in I_{0}$. So we have the contradiction with the fact that $(\bar{\nu}, \bar{\lambda}) \neq 0$.

Let us notice that

$$
\left\{\begin{array}{l}
d(x)=\frac{\partial H}{\partial u}(x, \bar{y}(x), \bar{u}(x), \bar{\varphi}(x)) \\
\frac{\partial \mathcal{L}}{\partial u}(\bar{u}, \bar{\lambda}) h=\int_{\Omega} \frac{\partial H}{\partial u}(x, \bar{y}(x), \bar{u}(x), \bar{\varphi}(x)) h(x) d x .
\end{array}\right.
$$

As an immediate consequence of Pontryagin's principle, Theorem 3.1, and Remark 3.7, we obtain the necessary first and second order optimality conditions as follows.

Corollary 4.2. Suppose that $\bar{u}$ is a local solution for problem $(\mathbf{P})$. Suppose also that assumptions (A1)-(A3) and the regularity assumption (3.1) hold. Then there exist real numbers $\bar{\lambda}_{j}, j=1, \ldots, n_{i}+n_{e}$, and functions $\bar{y} \in W^{1, p}(\Omega), \bar{\varphi} \in$ $W^{1, \min \left\{s^{\prime}, p\right\}}(\Omega)$ such that (4.1)-(4.3) hold as well as the following relations:

(4.8) $\frac{\partial H}{\partial u}(x, \bar{y}(x), \bar{u}(x), \bar{\varphi}(x))(k-\bar{u}(x)) \geq 0$ for all $u_{a}(x) \leq k \leq u_{b}(x)$, for a.e. $x \in \Omega$,

$$
\frac{\partial^{2} \mathcal{L}}{\partial u^{2}}(\bar{u}, \bar{\lambda}) h^{2} \geq 0 \text { for all } h \in C_{\bar{u}, L^{2}(\Omega)}^{0},
$$


and

$$
\frac{\partial^{2} H}{\partial u^{2}}(x, \bar{y}(x), \bar{u}(x), \bar{\varphi}(x)) \geq 0 \text { for a.e. } x \in \Omega \backslash \Omega^{0} .
$$

Let us notice that

$$
\frac{\partial H}{\partial u}(x, \bar{y}(x), \bar{u}(x), \bar{\varphi}(x))=d(x)=0, \quad x \in \Omega \backslash \Omega^{0} .
$$

Then it is enough to use elementary calculus to deduce (4.10) from (4.4) and the above equality.

In finite dimension, the first order optimality conditions and the strict positivity of the second derivative of the Lagrangian with respect to $u$ on $C_{\bar{u}}^{0}$ are sufficient conditions for a local minimum. The argument of the proof uses in an essential way the compactness of the balls in finite dimension. To extend this argumentation to infinitedimensional optimization problems, Bonnans and Zidani [2] made the assumption that the second derivative of the Lagrangian with respect to $u$ was a Legendre form. Let us recall that a quadratic form $Q$ on a Hilbert space $X$ is said to be a Legendre form if it is weakly lower semicontinuous, and for every sequence $\left\{x_{k}\right\} \subset X$ that converges weakly $x_{k} \rightarrow x$ and such that $Q\left(x_{k}\right) \rightarrow Q(x)$, we have that $x_{k} \rightarrow x$ strongly. Unfortunately this assumption is not fulfilled, in general, in the context of control theory. We follow a different approach to achieve the same result. Along with the strict positivity of the second derivative of the Lagrangian, we assume that the second derivative of the Hamiltonian with respect to $u$ is strictly positive on $\Omega \backslash \Omega^{\tau}$, for $\tau>0$, which is not far from the necessary condition provided in (4.10). More precisely, we have the following result.

TheOREM 4.3. Let $\bar{u}$ be an admissible control for problem $(\mathbf{P})$ satisfying (A1)(A3), the regularity assumption (3.1), and (4.1)-(4.4) for some $\bar{\lambda}_{j}, j=1, \ldots, n_{i}+n_{e}$. Let us suppose also that there exist $\omega>0$ and $\tau>0$ such that

$$
\left\{\begin{array}{l}
\frac{\partial^{2} H}{\partial u^{2}}(x, \bar{y}(x), \bar{u}(x), \bar{\varphi}(x)) \geq \omega \text { for a.e. } x \in \Omega \backslash \Omega^{\tau}, \\
\frac{\partial^{2} \mathcal{L}}{\partial u^{2}}(\bar{u}, \bar{\lambda}) h^{2}>0 \text { for all } h \in C_{\bar{u}, L^{2}(\Omega)}^{0} \backslash\{0\} .
\end{array}\right.
$$

Then there exist $\varepsilon>0$ and $\alpha>0$ such that $J(\bar{u})+\alpha\|u-\bar{u}\|_{L^{2}(\Omega)}^{2} \leq J(u)$ for all admissible control $u$ with $\|u-\bar{u}\|_{L^{\infty}(\Omega)} \leq \varepsilon$.

Proof. We will argue by contradiction. The proof is divided into five steps.

(i) Definition of a sequence $\left\{h_{k}\right\}$ of the unit sphere of $L^{2}(\Omega)$ converging weakly to $h$. Let us suppose that the theorem is false. Then there exists a sequence $\left\{u_{k}\right\}$ of admissible controls with $u_{k} \rightarrow \bar{u}$ in $L^{\infty}(\Omega)$ such that

$$
J(\bar{u})+\frac{1}{k}\left\|u_{k}-\bar{u}\right\|_{L^{2}(\Omega)}^{2}>J\left(u_{k}\right) .
$$

Let us set $\delta_{k}=\left\|u_{k}-\bar{u}\right\|_{L^{2}(\Omega)}$ and

$$
h_{k}=\frac{u_{k}-\bar{u}}{\delta_{k}} .
$$

Since $\left\|h_{k}\right\|_{L^{2}(\Omega)}=1$ for every $k$, there exists a subsequence of $\left\{h_{k}\right\}$, which will be denoted in the same way, and $h \in L^{2}(\Omega)$ such that $h_{k} \rightarrow h$ weakly in $L^{2}(\Omega)$. 
(ii) $\frac{\partial \mathcal{L}}{\partial u}(\bar{u}, \bar{\lambda}) h=0$. Let us denote $y_{k}=y_{u_{k}}$. Since $u_{k}$ is admissible, we have that

$$
F_{j}\left(y_{k}\right)=0 \text { if } 1 \leq j \leq n_{e}
$$

and

$$
F_{j}\left(y_{k}\right) \leq 0 \text { if } n_{e}+1 \leq j \leq n_{e}+n_{i} .
$$

Since $\bar{\lambda}_{j} \geq 0$ if $n_{e}+1 \leq j \leq n_{e}+n_{i}$, we have that

$$
\bar{\lambda}_{j} F_{j}\left(y_{k}\right) \leq 0 \text { for } 1 \leq j \leq n_{e}+n_{i} .
$$

On the other hand $\bar{\lambda}_{j} F_{j}(\bar{y})=0$. Hence (4.12) implies

$$
\mathcal{L}(\bar{u}, \bar{\lambda})+\frac{1}{k}\left\|u_{k}-\bar{u}\right\|_{L^{2}(\Omega)}^{2}>\mathcal{L}\left(u_{k}, \bar{\lambda}\right) .
$$

Moreover, $h$ satisfies the sign condition in (3.10), because every $h_{k}$ satisfies it, and the set of functions that satisfy the sign condition in (3.10) is convex and closed in $L^{2}(\Omega)$, and therefore weakly closed. Furthermore

$$
\mathcal{L}\left(u_{k}, \bar{\lambda}\right)=\mathcal{L}(\bar{u}, \bar{\lambda})+\delta_{k} \frac{\partial \mathcal{L}}{\partial u}\left(v_{k}, \bar{\lambda}\right) h_{k},
$$

where $v_{k}$ is an intermediate point between $\bar{u}$ and $u_{k}$. Using (4.13) and that $\delta_{k}>0$, we have that

$$
\frac{\partial \mathcal{L}}{\partial u}\left(v_{k}, \bar{\lambda}\right) h_{k}<\frac{1}{k \delta_{k}}\left\|u_{k}-\bar{u}\right\|_{L^{2}(\Omega)}^{2}=\frac{1}{k}\left\|u_{k}-\bar{u}\right\|_{L^{2}(\Omega)} .
$$

This expression can be written as follows:

$$
\int_{\Omega}\left(\frac{\partial L}{\partial u}\left(x, y_{v_{k}}, v_{k}\right)+\varphi_{v_{k}} \frac{\partial f}{\partial u}\left(x, y_{v_{k}}, v_{k}\right)\right) h_{k} d x<\frac{1}{k}\left\|u_{k}-\bar{u}\right\|_{L^{2}(\Omega)},
$$

where $y_{v_{k}}$ and $\varphi_{v_{k}}$ are, respectively, the state and adjoint state associated to $v_{k}$. The conditions imposed on $F_{j}$ and the uniform convergence $v_{k} \rightarrow \bar{u}$ imply the convergences $y_{v_{k}} \rightarrow \bar{y}$ uniformly and $\varphi_{v_{k}} \rightarrow \bar{\varphi}$ in $L^{2}(\Omega)$. Using (A1), (A2), and the weak convergence $h_{k} \rightarrow h$ in $L^{2}(\Omega)$, we can pass to the limit in (4.14) and obtain

$$
\frac{\partial \mathcal{L}}{\partial u}(\bar{u}, \bar{\lambda}) h \leq 0 .
$$

On the other hand, from (4.7), (4.8), and $h_{k}=\left(u_{k}-\bar{u}\right) / \delta_{k}$, with $\delta_{k}>0$ and $u_{a} \leq$ $u_{k} \leq u_{b}$, we get

$$
\frac{\partial \mathcal{L}}{\partial u}(\bar{u}, \bar{\lambda}) h_{k} \geq 0
$$

Taking the limit we obtain

$$
\frac{\partial \mathcal{L}}{\partial u}(\bar{u}, \bar{\lambda}) h \geq 0
$$

So (4.15) and (4.16) lead to

$$
\frac{\partial \mathcal{L}}{\partial u}(\bar{u}, \bar{\lambda}) h=0
$$


(iii) $h \in C_{\bar{u}, L^{2}(\Omega)}^{0}$. First we check that

$$
F_{j}^{\prime}(\bar{y}) z_{h}=0 \text { if }\left\{\begin{array}{l}
j \leq n_{e} \\
\text { or } \\
j>n_{e}, F_{j}(\bar{y})=0, \bar{\lambda}_{j}>0
\end{array}\right.
$$

and

$$
F_{j}^{\prime}(\bar{y}) z_{h} \leq 0 \text { if } j>n_{e}, F_{j}(\bar{y})=0, \bar{\lambda}_{j}=0 .
$$

If $j \leq n_{e}$, then $F_{j}\left(y_{k}\right)=F_{j}\left(y_{\bar{u}+\delta_{k} h_{k}}\right)=0$ and $F_{j}(\bar{y})=0$. Therefore

$$
0=\frac{F_{j}\left(y_{\bar{u}+\delta_{k} h_{k}}\right)-F_{j}(\bar{y})}{\delta_{k}},
$$

and taking the limit we obtain with the help of assumption (A3)

$$
F_{j}^{\prime}(\bar{y}) z_{h}=0
$$

If $j>n_{e}$ and $F_{j}(\bar{y})=0$, we have that $F_{j}\left(y_{k}\right)=F_{j}\left(y_{\bar{u}+\delta_{k} h_{k}}\right) \leq 0$. So

$$
0 \geq \frac{F_{j}\left(y_{\bar{u}+\delta_{k} h_{k}}\right)-F_{j}(\bar{y})}{\delta_{k}},
$$

and once again taking the limit as before we deduce

$$
F_{j}^{\prime}(\bar{y}) z_{h} \leq 0 \text {. }
$$

Let us see what happens when $\bar{\lambda}_{j}>0$. Taking into account (4.12) and that $\delta_{k}=$ $\left\|u_{k}-\bar{u}\right\|_{L^{2}(\Omega)}$, we get

$$
\frac{\delta_{k}}{k} \geq \frac{J\left(u_{k}\right)-J(\bar{u})}{\delta_{k}}=\frac{J\left(\bar{u}+\delta_{k} h_{k}\right)-J(\bar{u})}{\delta_{k}} .
$$

Since $\delta_{k} \rightarrow 0$, by passing to the limit in this expression, it follows that

$$
0 \geq J^{\prime}(\bar{u}) h .
$$

Using (4.17) and the expression for the derivative of the Lagrangian, we now have that

$$
0=J^{\prime}(\bar{u}) h+\sum_{j=1}^{n_{e}+n_{i}} \bar{\lambda}_{j} F_{j}^{\prime}(\bar{y}) z_{h} .
$$

Taking into account that if $j \leq n_{e}$, then we have already proved the equalities $F_{j}^{\prime}(\bar{y}) z_{h}=0$, and that if $F_{j}(\bar{y})<0$, then $\bar{\lambda}_{j}=0$, we have that

$$
0=J^{\prime}(\bar{u}) h+\sum_{j \in I_{1}} \bar{\lambda}_{j} F_{j}^{\prime}(\bar{y}) z_{h},
$$

where

$$
I_{1}=\left\{j: n_{e}<j \leq n_{e}+n_{i} ; F_{j}(\bar{y})=0 ; \bar{\lambda}_{j}>0\right\} .
$$


So

$$
0 \leq-J^{\prime}(\bar{u}) h=\sum_{j \in I_{1}} \bar{\lambda}_{j} F_{j}^{\prime}(\bar{y}) z_{h} \leq 0
$$

Thus, if $j \in I_{1}$, then necessarily $F_{j}^{\prime}(\bar{y}) z_{h}=0$. To conclude the proof of the inclusion $h \in C_{\bar{u}, L^{2}(\Omega)}^{0}$ it remains to check that $h(x)=0$ for a.e. $x \in \Omega^{0}$. As signaled above, $h$ satisfies the sign condition; then we have that $d(x) h(x) \geq 0$ for a.e. $x \in \Omega$; recall (3.7). Therefore

$$
\int_{\Omega}|d(x) h(x)| d x=\int_{\Omega} d(x) h(x) d x=\frac{\partial \mathcal{L}}{\partial u}(\bar{u}, \bar{\lambda}) h=0,
$$

which implies $h(x)=0$ in a.e. $\Omega^{0}$ and $h \in C_{\bar{u}, L^{2}(\Omega)}^{0}$.

(iv) $h=0$. Due to the assumption of the theorem, we have that

$$
\frac{\partial^{2} \mathcal{L}}{\partial u^{2}}(\bar{u}, \bar{\lambda}) h^{2}>0 \text { if } h \neq 0
$$

Let us prove that the reverse inequality is satisfied, which will lead to the identity $h=0$. By applying the mean value theorem we get

$$
\mathcal{L}\left(u_{k}, \bar{\lambda}\right)=\mathcal{L}(\bar{u}, \bar{\lambda})+\delta_{k} \frac{\partial \mathcal{L}}{\partial u}(\bar{u}, \bar{\lambda}) h_{k}+\frac{\delta_{k}^{2}}{2} \frac{\partial^{2} \mathcal{L}}{\partial u^{2}}\left(w_{k}, \bar{\lambda}\right) h_{k}^{2},
$$

where $w_{k}$ is an intermediate point between $u_{k}$ and $\bar{u}$. In order to simplify the expression of the derivatives of $\mathcal{L}$, let us introduce some notation:

$$
\begin{gathered}
\bar{H}_{u}(x)=\frac{\partial H}{\partial u}(x, \bar{y}(x), \bar{u}(x), \bar{\varphi}(x)), \\
\bar{H}_{u u}(x)=\frac{\partial^{2} H}{\partial u^{2}}(x, \bar{y}(x), \bar{u}(x), \bar{\varphi}(x)) .
\end{gathered}
$$

Analogously we define $\bar{H}_{u y}$ or $\bar{H}_{y y}$. Inserting this notation into the expressions of the derivatives of $\mathcal{L}$ given in (3.5) and (3.12), we get

$$
\begin{aligned}
& \delta_{k} \frac{\partial \mathcal{L}}{\partial u}(\bar{u}, \bar{\lambda}) h_{k}+\frac{\delta_{k}^{2}}{2} \frac{\partial^{2} \mathcal{L}}{\partial u^{2}}(\bar{u}, \bar{\lambda}) h_{k}^{2}=\delta_{k} \int_{\Omega} \bar{H}_{u}(x) h_{k}(x) d x+\frac{\delta_{k}^{2}}{2} \int_{\Omega} \bar{H}_{u u}(x) h_{k}^{2}(x) d x \\
& +\frac{\delta_{k}^{2}}{2}\left[\int_{\Omega} \bar{H}_{y y}(x) z_{h_{k}}^{2}(x) d x+2 \int_{\Omega} \bar{H}_{y u}(x) h_{k}(x) z_{h_{k}}(x) d x+\sum_{j=1}^{n_{e}+n_{i}} \bar{\lambda}_{j} F_{j}^{\prime \prime}(\bar{y}) z_{h_{k}}^{2}\right] .
\end{aligned}
$$

Taking into account that $\bar{H}_{u}(x)=d(x)=0$ in $\Omega \backslash \Omega^{0}$,

$$
\begin{aligned}
& A_{k}=\delta_{k} \int_{\Omega} \bar{H}_{u}(x) h_{k}(x) d x+\frac{\delta_{k}^{2}}{2} \int_{\Omega} \bar{H}_{u u}(x) h_{k}^{2}(x) d x=\delta_{k} \int_{\Omega^{0} \backslash \Omega^{\tau}} \bar{H}_{u}(x) h_{k}(x) d x \\
& +\delta_{k} \int_{\Omega^{\tau}} \bar{H}_{u}(x) h_{k}(x) d x+\frac{\delta_{k}^{2}}{2} \int_{\Omega^{\tau}} \bar{H}_{u u}(x) h_{k}^{2}(x) d x+\frac{\delta_{k}^{2}}{2} \int_{\Omega \backslash \Omega^{\tau}} \bar{H}_{u u}(x) h_{k}^{2}(x) d x
\end{aligned}
$$


Using now that $\bar{H}_{u}(x) h_{k}(x) \geq 0$ for a.e. $x \in \Omega$ and $\bar{H}_{u}(x) \geq \tau$ for a.e. $x \in \Omega^{\tau}$, we have that

$$
A_{k} \geq \delta_{k} \tau \int_{\Omega^{\tau}}\left|h_{k}(x)\right| d x+\frac{\delta_{k}^{2}}{2} \int_{\Omega^{\tau}} \bar{H}_{u u}(x) h_{k}^{2}(x) d x+\frac{\delta_{k}^{2}}{2} \int_{\Omega \backslash \Omega^{\tau}} \bar{H}_{u u}(x) h_{k}^{2}(x) d x .
$$

Since $\left\|\delta_{k} h_{k}\right\|_{L^{\infty}(\Omega)}=\left\|u_{k}-\bar{u}\right\|_{L^{\infty}(\Omega)}<\varepsilon$, then for a.e. $x \in \Omega, \delta_{k}\left|h_{k}(x)\right| \leq \varepsilon$. Therefore

$$
\frac{\delta_{k}^{2} h_{k}^{2}(x)}{\varepsilon} \leq \delta_{k}\left|h_{k}(x)\right| \text {. }
$$

Hence

$$
A_{k} \geq \frac{\delta_{k}^{2}}{2} \int_{\Omega^{\tau}}\left(\frac{2 \tau}{\varepsilon}+\bar{H}_{u u}(x)\right) h_{k}^{2}(x) d x+\frac{\delta_{k}^{2}}{2} \int_{\Omega \backslash \Omega^{\tau}} \bar{H}_{u u}(x) h_{k}^{2}(x) d x .
$$

Now, from (4.13), (4.19) and taking into account the previous considerations, we have

$$
\begin{gathered}
\frac{\delta_{k}^{2}}{k}>\delta_{k} \frac{\partial \mathcal{L}}{\partial u}(\bar{u}, \bar{\lambda}) h_{k}+\frac{\delta_{k}^{2}}{2} \frac{\partial^{2} \mathcal{L}}{\partial u^{2}}\left(w_{k}, \bar{\lambda}\right) h_{k}^{2} \\
=\delta_{k} \frac{\partial \mathcal{L}}{\partial u}(\bar{u}, \bar{\lambda}) h_{k}+\frac{\delta_{k}^{2}}{2} \frac{\partial^{2} \mathcal{L}}{\partial u^{2}}(\bar{u}, \bar{\lambda}) h_{k}^{2}+\frac{\delta_{k}^{2}}{2}\left[\frac{\partial^{2} \mathcal{L}}{\partial u^{2}}\left(w_{k}, \bar{\lambda}\right) h_{k}^{2}-\frac{\partial^{2} \mathcal{L}}{\partial u^{2}}(\bar{u}, \bar{\lambda}) h_{k}^{2}\right] \\
\geq \frac{\delta_{k}^{2}}{2} \int_{\Omega^{\tau}}\left(\frac{2 \tau}{\varepsilon}+\bar{H}_{u u}(x)\right) h_{k}^{2}(x) d x+\frac{\delta_{k}^{2}}{2} \int_{\Omega \backslash \Omega^{\tau}} \bar{H}_{u u}(x) h_{k}^{2}(x) d x \\
+\frac{\delta_{k}^{2}}{2}\left[\int_{\Omega} \bar{H}_{y y}(x) z_{h_{k}}^{2}(x) d x+2 \int_{\Omega} \bar{H}_{y u}(x) h_{k}(x) z_{h_{k}}(x) d x+\sum_{j=1}^{n_{e}+n_{i}} \bar{\lambda}_{j} F^{\prime \prime}(\bar{y}) z_{h_{k}}^{2}\right] \\
+\frac{\delta_{k}^{2}}{2}\left[\frac{\partial^{2} \mathcal{L}}{\partial u^{2}}\left(w_{k}, \bar{\lambda}\right) h_{k}^{2}-\frac{\partial^{2} \mathcal{L}}{\partial u^{2}}(\bar{u}, \bar{\lambda}) h_{k}^{2}\right] .
\end{gathered}
$$

Taking into account the assumptions made on the second derivatives of the functions, there exists a constant $C_{H}>0$ such that $\bar{H}_{u u}(x) \geq-C_{H}$ for a.e. $x \in \Omega$. So, taking $\varepsilon$ small enough, we have that

$$
\frac{2 \tau}{\varepsilon}+\bar{H}_{u u}(x) \geq \frac{2 \tau}{\varepsilon}-C_{H}>0 \text { for a.e. } x \in \Omega .
$$

Thus

$$
\liminf _{k \rightarrow \infty} \int_{\Omega^{\tau}}\left(\frac{2 \tau}{\varepsilon}+\bar{H}_{u u}(x)\right) h_{k}^{2}(x) d x \geq \int_{\Omega^{\tau}}\left(\frac{2 \tau}{\varepsilon}+\bar{H}_{u u}(x)\right) h^{2}(x) d x .
$$

Moreover, in $\Omega \backslash \Omega^{\tau}, \bar{H}_{u u}(x)>\omega>0$, and then

$$
\liminf _{k \rightarrow \infty} \int_{\Omega \backslash \Omega^{\tau}} \bar{H}_{u u}(x) h_{k}^{2}(x) d x \geq \int_{\Omega \backslash \Omega^{\tau}} \bar{H}_{u u}(x) h^{2}(x) d x .
$$

Now dividing (4.20) by $\delta_{k}^{2} / 2$ and using (3.14) and assumption (A3), we can take the lower limit of the resulting expression and obtain

$$
\begin{gathered}
0 \geq \int_{\Omega^{\tau}}\left(\frac{2 \tau}{\varepsilon}+\bar{H}_{u u}(x)\right) h^{2}(x) d x+\int_{\Omega \backslash \Omega^{\tau}} \bar{H}_{u u}(x) h^{2}(x) d x \\
+\int_{\Omega} \bar{H}_{y y}(x) z_{h}^{2}(x) d x+2 \int_{\Omega} \bar{H}_{y u}(x) h(x) z_{h}(x) d x+\sum_{j=1}^{n_{e}+n_{i}} \bar{\lambda}_{j} F_{j}^{\prime \prime}(\bar{y}) z_{h}^{2} \\
=\frac{2 \tau}{\varepsilon} \int_{\Omega} h^{2}(x) d x+\frac{\partial^{2} \mathcal{L}}{\partial u^{2}}(\bar{u}, \bar{\lambda}) h^{2} .
\end{gathered}
$$


Combining this inequality with (4.18) we deduce that $h=0$.

(v) $h_{k} \rightarrow 0$ strongly in $L^{2}(\Omega)$. We have that $h_{k} \rightarrow h=0$ weakly in $L^{2}(\Omega)$, and consequently $z_{h_{k}} \rightarrow 0$ strongly in $W^{1, q}(\Omega)$. Therefore again dividing (4.20) by $\delta_{k}^{2} / 2$ and using (3.14) we get

$$
\begin{gathered}
\min \left\{\omega, \frac{2 \tau}{\varepsilon}-C_{H}\right\} \limsup _{k \rightarrow \infty} \int_{\Omega} h_{k}^{2}(x) d x \\
\leq \limsup _{k \rightarrow \infty}\left\{\int_{\Omega^{\tau}}\left(\frac{2 \tau}{\varepsilon}+\bar{H}_{u u}(x)\right) h_{k}^{2}(x) d x+\int_{\Omega \backslash \Omega^{\tau}} \bar{H}_{u u}(x) h_{k}^{2}(x) d x\right\} \\
\leq \limsup _{k \rightarrow \infty}\left\{\frac{1}{k}-\left[\int_{\Omega} \bar{H}_{y y}(x) z_{h_{k}}^{2}(x) d x+2 \int_{\Omega} \bar{H}_{y u}(x) h_{k}(x) z_{h_{k}}(x) d x+\sum_{j=1}^{n_{e}+n_{i}} \bar{\lambda}_{j} F^{\prime \prime}(\bar{y}) z_{h_{k}}^{2}\right]\right. \\
\left.-\left[\frac{\partial^{2} \mathcal{L}}{\partial u^{2}}\left(w_{k}, \bar{\lambda}\right) h_{k}^{2}-\frac{\partial^{2} \mathcal{L}}{\partial u^{2}}(\bar{u}, \bar{\lambda}) h_{k}^{2}\right]\right\}=0 .
\end{gathered}
$$

Hence

$$
\lim _{k \rightarrow \infty}\left\|h_{k}\right\|_{L^{2}(\Omega)}=0 .
$$

But $\left\|h_{k}\right\|_{L^{2}(\Omega)}=1$ for every $k$. So we have achieved the contradiction.

The next theorem shows the equivalence of (3.20) and (4.11).

THEOREM 4.4. Let $\bar{u}$ be an admissible control for problem $(\mathbf{P})$ that satisfies (A1)-(A3), the regularity assumption (3.1), and (4.1)-(4.4). Then the following two statements are equivalent:

(1) There exist $\delta>0$ and $\tau^{\prime}>0$ such that

$$
\frac{\partial^{2} \mathcal{L}}{\partial u^{2}}(\bar{u}, \bar{\lambda}) h^{2} \geq \delta\|h\|_{L^{2}(\Omega)}^{2} \text { for all } h \in C_{\bar{u}, L^{2}(\Omega)}^{\tau^{\prime}} .
$$

(2) There exist $\omega>0$ and $\tau>0$ such that

$$
\left\{\begin{array}{l}
\frac{\partial^{2} H}{\partial u^{2}}(x, \bar{y}(x), \bar{u}(x), \bar{\varphi}(x)) \geq \omega \text { for a.e. } x \in \Omega \backslash \Omega^{\tau}, \\
\frac{\partial^{2} \mathcal{L}}{\partial u^{2}}(\bar{u}, \bar{\lambda}) h^{2}>0 \text { for all } h \in C_{\bar{u}, L^{2}(\Omega)}^{0} \backslash\{0\} .
\end{array}\right.
$$

Proof. (1) $\Longrightarrow(2)$. Since $C_{\bar{u}, L^{2}(\Omega)}^{0} \subset C_{\bar{u}, L^{2}(\Omega)}^{\tau^{\prime}}$, the second inequality of (4.22) is an obvious consequence of (4.21). Let us prove the existence of $\omega$ and $\tau$ satisfying the first inequality of (4.22). Let us take $\alpha>0$ and $\varepsilon>0$, as in Theorem 3.5, and consider the problem

$$
\left(P_{\alpha}\right)\left\{\begin{array}{l}
\text { Minimize } J_{\alpha}(u)=J(u)-\frac{\alpha}{2}\|u-\bar{u}\|_{L^{2}(\Omega)}^{2}, \\
u_{a}(x) \leq u(x) \leq u_{b}(x) \text { for a.e. } x \in \Omega \\
G_{j}(u)=0,1 \leq j \leq n_{e} \\
G_{j}(u) \leq 0, n_{e}+1 \leq j \leq n_{e}+n_{i} .
\end{array}\right.
$$

Then for any feasible point $u$ of this problem, with $\|u-\bar{u}\|_{\infty}<\varepsilon$ and $u \neq \bar{u}$, we have

$$
J_{\alpha}(\bar{u})=J(\bar{u}) \leq J(u)-\alpha\|u-\bar{u}\|_{L^{2}(\Omega)}^{2}<J(u)-\frac{\alpha}{2}\|u-\bar{u}\|_{L^{2}(\Omega)}^{2}=J_{\alpha}(u) .
$$


Then $\bar{u}$ is the unique solution of $\left(P_{\alpha}\right)$ in the $L^{\infty}(\Omega)$-ball $B_{\varepsilon}(\bar{u})$. The Hamiltonian for problem $\left(\mathrm{P}_{\alpha}\right)$ is

$$
H^{\alpha}(x, y, u, \varphi)=H(x, y, u, \varphi)-\frac{\alpha}{2}(u-\bar{u}(x))^{2} .
$$

Therefore we can apply Corollary 4.2 to $\left(P_{\alpha}\right)$ and deduce, with the notation of the proof of Theorem 4.3, that

$$
\bar{H}_{u u}(x)-\alpha=\bar{H}_{u u}^{\alpha}(x) \geq 0 \text { for a.e. } x \in \Omega \backslash \Omega^{0},
$$

which implies

$$
\bar{H}_{u u}(x) \geq \alpha>0 \text { for a.e. } x \in \Omega \backslash \Omega^{0} .
$$

In the case in which the bound constraints on the control are not active, i.e., $u_{a}(x)<\bar{u}(x)<u_{b}(x)$ a.e., then the Lebesgue measure of $\Omega^{0}$ is zero; hence (4.23) implies the first inequality of (4.22). Let us analyze the case where $\Omega^{0}$ has a strictly positive Lebesgue measure. We will proceed by contradiction and we assume that there exist no $\omega>0$ and $\tau>0$ such that (4.22) is satisfied. Then we define for every $k \geq 1$

$$
\hat{h}_{k}(x)=\left\{\begin{array}{cl}
+1 & \text { if }|d(x)| \leq 1 / k, \bar{H}_{u u}(x)<1 / k, \text { and } \bar{u}(x)=u_{a}(x), \\
-1 & \text { if }|d(x)| \leq 1 / k, \bar{H}_{u u}(x)<1 / k, \text { and } \bar{u}(x)=u_{b}(x), \\
0 & \text { otherwise. }
\end{array}\right.
$$

Since (4.22) is not satisfied for $\omega=1 / k$ and $\tau=1 / k$, with arbitrarily large $k$, and the measure of $\Omega^{0}$ is not zero, we have that $\hat{h}_{k} \neq 0$. Then we define $\tilde{h}_{k}=\hat{h}_{k} /\left\|\hat{h}_{k}\right\|_{L^{2}(\Omega)}$. Let us prove that $\tilde{h}_{k} \rightarrow 0$ weakly in $L^{2}(\Omega)$. From (4.23) we deduce that the set

$$
B=\left\{x \in \Omega:|d(x)|=0 \text { and } \bar{H}_{u u}(x) \leq 0\right\}
$$

has zero Lebesgue measure. Therefore

$$
\bigcap_{k=1}^{\infty} \operatorname{supp}\left\{\tilde{h}_{k}\right\} \subset B \Rightarrow \operatorname{measure}\left(\bigcap_{k=1}^{\infty} \operatorname{supp}\left\{\tilde{h}_{k}\right\}\right) \leq \operatorname{measure}(B)=0 .
$$

Taking into account that $\operatorname{supp}\left\{\tilde{h}_{k}\right\} \subset \operatorname{supp}\left\{\tilde{h}_{k^{\prime}}\right\}$ for every $k>k^{\prime}$, we deduce that $\tilde{h}_{k}(x) \rightarrow 0$ pointwise a.e. in $\Omega$. On the other hand, $\left\{\tilde{h}_{k}\right\}_{k=1}^{\infty}$ is bounded in $L^{2}(\Omega)$; consequently $\tilde{h}_{k} \rightarrow 0$ weakly in $L^{2}(\Omega)$; see Hewitt and Stromberg [14, p. 207]. Furthermore for $\tau^{\prime}>1 / k$ we have that $\tilde{h}_{k}(x)=0$ for every $x \in \Omega^{\tau^{\prime}}$ and $\tilde{h}_{k}$ satisfies the sign condition of (3.10). Let us define a new function $h_{k} \in C_{\bar{u}, L^{2}(\Omega)}^{\tau^{\prime}}$ close to $\tilde{h}_{k}$. Using the functions $\left\{\bar{h}_{j}\right\}_{j \in I_{0}}$ introduced in (3.1), we set

$$
h_{k}=\tilde{h}_{k}-\sum_{j \in I_{0}} \alpha_{k j} \bar{h}_{j}, \quad \text { with } \alpha_{k j}=G_{j}^{\prime}(\bar{u}) \tilde{h}_{k} .
$$

As in the proof of Proposition 3.6, we deduce that $h_{k} \in C_{\bar{u}, L^{2}(\Omega)}^{\tau^{\prime}}$ for every $k>1 / \tau^{\prime}$. Moreover, since $\tilde{h}_{k} \rightarrow 0$ weakly in $L^{2}(\Omega)$, we deduce that $\alpha_{k j} \rightarrow 0$, and therefore $h_{k} \rightarrow 0$ weakly in $L^{2}(\Omega)$. On the other hand, since $\operatorname{supp}\left\{\tilde{h}_{k}\right\}$ is included in the set 
of points of $\Omega$ where the bound constraints on the control are active, which has an empty intersection with the support of each $\bar{h}_{j}\left(j \in I_{0}\right)$,

$$
\begin{gathered}
\left\|h_{k}\right\|_{L^{2}(\Omega)}=\left\{\int_{\operatorname{supp}\left\{\tilde{h}_{k}\right\}} h_{k}^{2} d x+\int_{\Omega \backslash \operatorname{supp}\left\{\tilde{h}_{k}\right\}} h_{k}^{2} d x\right\}^{\frac{1}{2}} \\
=\left\{\int_{\operatorname{supp}\left\{\tilde{h}_{k}\right\}} \tilde{h}_{k}^{2} d x+\int_{\Omega \backslash \operatorname{supp}\left\{\tilde{h}_{k}\right\}}\left(\sum_{j \in I_{0}} \alpha_{k j} \bar{h}_{j}\right)^{2} d x\right\}^{\frac{1}{2}} \\
=\left\{\left\|\tilde{h}_{k}\right\|_{L^{2}(\Omega)}^{2}+\left\|\sum_{j \in I_{0}} \alpha_{k j} \bar{h}_{j}\right\|_{L^{2}(\Omega)}^{2}\right\}^{\frac{1}{2}} \geq\left\{1-2 \sum_{j \in I_{0}} \alpha_{k j}^{2}\left\|\bar{h}_{j}\right\|_{L^{2}(\Omega)}^{2}\right\}^{\frac{1}{2}} \stackrel{k \rightarrow \infty}{\longrightarrow} 1 .
\end{gathered}
$$

From this relation and (4.21) with $h=h_{k}$, we get

$$
\delta \leq \delta \liminf _{k \rightarrow \infty}\left\|h_{k}\right\|_{L^{2}(\Omega)}^{2} \leq \liminf _{k \rightarrow \infty} \frac{\partial^{2} \mathcal{L}}{\partial u^{2}}(\bar{u}, \bar{\lambda}) h_{k}^{2} .
$$

On the other hand, the weak convergence $h_{k} \rightarrow 0$ in $L^{2}(\Omega)$ implies the strong convergence in $\left(W^{1, q^{\prime}}(\Omega)\right)^{\prime}$, and thanks to Lemma 2.4 we deduce $z_{h_{k}} \rightarrow 0$ in $W^{1, q}(\Omega) \subset$ $L^{2}(\Omega)$ strongly. Writing the second derivative of the Lagrangian in terms of the derivatives of the Hamiltonian, as was done in the proof of Theorem 4.3, and taking into account that $\bar{H}_{u u}(x)<1 / k$ in the support of $\tilde{h}_{k}$ and $\alpha_{k j} \rightarrow 0$, we get

$$
\begin{gathered}
\liminf _{k \rightarrow \infty} \frac{\partial^{2} \mathcal{L}}{\partial u^{2}}(\bar{u}, \bar{\lambda}) h_{k}^{2} \leq \limsup _{k \rightarrow \infty} \int_{\Omega} \bar{H}_{u u}(x) h_{k}^{2}(x) d x+\limsup _{k \rightarrow \infty} \int_{\Omega} \bar{H}_{y y}(x) z_{h_{k}}^{2}(x) d x \\
+2 \limsup _{k \rightarrow \infty} \int_{\Omega} \bar{H}_{y u}(x) h_{k}(x) z_{h_{k}}(x) d x+\limsup _{k \rightarrow \infty}\left(\sum_{j=1}^{n_{e}+n_{i}} \bar{\lambda}_{j} F_{j}^{\prime \prime}(\bar{y}) z_{h_{k}}^{2}\right) \\
\leq \limsup _{k \rightarrow \infty} \int_{\operatorname{supp}\left\{\tilde{h}_{k}\right\}} \bar{H}_{u u}(x) h_{k}^{2}(x) d x+\limsup _{k \rightarrow \infty} \int_{\Omega \backslash \operatorname{supp}\left\{\tilde{h}_{k}\right\}} \bar{H}_{u u}(x) h_{k}^{2}(x) d x \\
\leq \limsup _{k \rightarrow \infty} \frac{1}{k} \int_{\operatorname{supp}\left\{\tilde{h}_{k}\right\}} \tilde{h}_{k}^{2}(x) d x+\limsup _{k \rightarrow \infty} \int_{\Omega \backslash \operatorname{supp}\left\{\tilde{h}_{k}\right\}} \bar{H}_{u u}(x)\left[\sum_{j \in I_{0}} \alpha_{k j} \bar{h}_{j}(x)\right]^{2} d x \\
=\limsup _{k \rightarrow \infty} \frac{1}{k} \int_{\Omega} \tilde{h}_{k}^{2}(x) d x=\lim _{k \rightarrow \infty} \frac{1}{k}=0,
\end{gathered}
$$

which contradicts (4.24).

$(2) \Longrightarrow(1)$. Let us suppose that $(4.21)$ is not satisfied. Then for every $\tau^{\prime}>0$ there exists $h_{\tau^{\prime}} \in C_{\bar{u}, L^{2}(\Omega)}^{\tau^{\prime}}$ such that $\left\|h_{\tau^{\prime}}\right\|_{L^{2}(\Omega)}=1$ and

$$
\frac{\partial^{2} \mathcal{L}}{\partial u^{2}}(\bar{u}, \bar{\lambda}) h_{\tau^{\prime}}^{2}<\tau^{\prime}
$$

Since $\left\{h_{\tau^{\prime}}\right\}$ is bounded in $L^{2}(\Omega)$, there exists a subsequence, denoted in the same way, such that $h_{\tau^{\prime}} \rightarrow h$ weakly in $L^{2}(\Omega)$. We have that $h \in C_{\bar{u}, L^{2}(\Omega)}^{0}$. Indeed relations (3.10) are obtained for $h$ by passing to the limit in the corresponding ones satisfied 
by $h_{\tau^{\prime}}$. Let us see that $h(x)=0$ in $\Omega^{0}$ :

$$
\begin{gathered}
\int_{\Omega}|h(x)||d(x)| d x=\int_{\Omega} h(x) d(x) d x=\lim _{\tau^{\prime} \rightarrow 0} \int_{\Omega} h_{\tau^{\prime}}(x) d(x) d x \\
=\lim _{\tau^{\prime} \rightarrow 0} \int_{\Omega \backslash \Omega^{\tau^{\prime}}}\left|h_{\tau^{\prime}}(x) \| d(x)\right| d x \\
\leq \lim _{\tau^{\prime} \rightarrow 0} \tau^{\prime} \int_{\Omega}\left|h_{\tau^{\prime}}(x)\right| d x \leq \lim _{\tau^{\prime} \rightarrow 0} \tau^{\prime} \sqrt{m(\Omega)}\left\|h_{\tau^{\prime}}\right\|_{L^{2}(\Omega)}=0
\end{gathered}
$$

hence $h(x) d(x)=0$, and therefore $h(x)=0$ for a.e. $x \in \Omega^{0}$.

Since $\bar{H}_{u u}(x) \geq \omega>0$ in $\Omega \backslash \Omega^{\tau},\left(\Omega \backslash \Omega^{\tau^{\prime}}\right) \subset\left(\Omega \backslash \Omega^{\tau}\right)$ for $\tau^{\prime}<\tau$, and $h_{\tau^{\prime}}=0$ in $\Omega_{\tau^{\prime}}$, we have that

$$
\begin{gathered}
\liminf _{k \rightarrow \infty} \int_{\Omega} \bar{H}_{u u}(x) h_{\tau^{\prime}}^{2}(x) d x=\liminf _{k \rightarrow \infty} \int_{\Omega \backslash \Omega^{\tau^{\prime}}} \bar{H}_{u u}(x) h_{\tau^{\prime}}^{2}(x) d x \\
\geq \int_{\Omega \backslash \Omega^{\tau^{\prime}}} \bar{H}_{u u}(x) h^{2}(x) d x=\int_{\Omega} \bar{H}_{u u}(x) h^{2}(x) d x .
\end{gathered}
$$

Therefore, using the definition of $h_{\tau^{\prime}}$ along with the strong convergence $z_{h_{\tau^{\prime}}} \rightarrow z_{h}$ in $W^{1, q}(\Omega)$, we get

$$
\begin{gathered}
0 \geq \limsup _{\tau^{\prime} \rightarrow 0} \frac{\partial^{2} \mathcal{L}}{\partial u^{2}}(\bar{u}, \bar{\lambda}) h_{\tau^{\prime}}^{2} \geq \liminf _{\tau^{\prime} \rightarrow 0} \frac{\partial^{2} \mathcal{L}}{\partial u^{2}}(\bar{u}, \bar{\lambda}) h_{\tau^{\prime}}^{2} \\
=\liminf _{\tau^{\prime} \rightarrow 0}\left\{\int_{\Omega} \bar{H}_{u u}(x) h_{\tau^{\prime}}^{2}(x) d x+\int_{\Omega} \bar{H}_{y y}(x) z_{h_{\tau^{\prime}}}^{2}(x) d x\right. \\
\left.+2 \int_{\Omega} \bar{H}_{y u}(x) h_{\tau^{\prime}}(x) z_{h_{\tau^{\prime}}}(x) d x+\sum_{j=1}^{n_{e}+n_{i}} \bar{\lambda}_{j} F_{j}^{\prime \prime}(\bar{y}) z_{h_{\tau^{\prime}}}^{2}\right\} \geq \frac{\partial^{2} \mathcal{L}}{\partial u^{2}}(\bar{u}, \bar{\lambda}) h^{2},
\end{gathered}
$$

which, together with (4.22), implies that $h=0$. Finally, using the weak convergence $h_{\tau^{\prime}} \rightarrow 0$ in $L^{2}(\Omega)$ and the strong convergence $z_{h_{\tau^{\prime}}} \rightarrow 0$ in $W^{1, q}(\Omega)$, we conclude that

$$
\begin{gathered}
\omega=\omega \limsup _{\tau^{\prime} \rightarrow 0}\left\|h_{\tau^{\prime}}\right\|_{L^{2}(\Omega)}^{2} \leq \limsup _{\tau^{\prime} \rightarrow 0} \int_{\Omega} \bar{H}_{u u}(x) h_{\tau^{\prime}}^{2}(x) d x \\
\leq \limsup _{\tau^{\prime} \rightarrow 0}\left\{\frac{\partial^{2} \mathcal{L}}{\partial u^{2}}(\bar{u}, \bar{\lambda}) h_{\tau^{\prime}}^{2} d x-\int_{\Omega} \bar{H}_{y y}(x) z_{h_{\tau^{\prime}}}^{2}(x) d x\right. \\
\left.-2 \int_{\Omega} \bar{H}_{y u}(x) h_{\tau^{\prime}}(x) z_{h_{\tau^{\prime}}}(x) d x-\sum_{j=1}^{n_{e}+n_{i}} \bar{\lambda}_{j} F_{j}^{\prime \prime}(\bar{y}) z_{h_{\tau^{\prime}}}^{2}\right\} \leq 0,
\end{gathered}
$$

and we have a contradiction.

\section{REFERENCES}

[1] J. Bonnans And E. CASAs, Contrôle de systèmes elliptiques semilinéaires comportant des contraintes sur l'état, in Nonlinear Partial Differential Equations and Their Applications, vol. 8, Collège de France Seminar, H. Brezis and J. Lions, eds., Longman Scientific and Technical, New York, 1988, pp. 69-86. 
[2] J. F. Bonnans And H. Zidani, Optimal control problems with partially polyhedric constraints, SIAM J. Control Optim., 37 (1999), pp. 1726-1741.

[3] H. Cartan, Calcul Différentiel, Hermann, Paris, 1967.

[4] E. CASAs, Pontryagin's principle for optimal control problems governed by semilinear elliptic equations, in International Conference on Control and Estimation of Distributed Parameter Systems: Nonlinear Phenomena, vol. 118, Internat. Ser. Numer. Math. 118, Birkhäuser, Basel, 1994, pp. 97-114.

[5] E. Casas, M. Mateos, And L. Fernández, Second-order optimality conditions for semilinear elliptic control problems with constraints on the gradient of the state, Control Cybernet., 28 (1999), pp. 463-479.

[6] E. Casas, J.-P. Raymond, And H. Zidani, Pontryagin's principle for local solutions of control problems with mixed control-state constraints, SIAM J. Control Optim., 39 (2000), pp. 1182-1203.

[7] E. Casas And F. Tröltzsch, Second order necessary and sufficient optimality conditions for optimization problems and applications to control theory, SIAM J. Optim., to appear.

[8] E. Casas and F. Tröltzsch, Second order necessary optimality conditions for some stateconstrained control problems of semilinear elliptic equations, Appl. Math. Optim., 39 (1999), pp. 211-227.

[9] E. Casas, F. Tröltzsch, And A. Unger, Second order sufficient optimality conditions for a nonlinear elliptic control problem, Z. Anal. Anwendungen, 15 (1996), pp. 687-707.

[10] E. Casas, F. Tröltzsch, And A. Unger, Second order sufficient optimality conditions for some state-constrained control problems of semilinear elliptic equations, SIAM J. Control Optim., 38 (2000), pp. 1369-1391.

[11] F. Clarke, A new approach to Lagrange multipliers, Math. Oper. Res., 1 (1976), pp. 165-174.

[12] J. C. DunN, On second order sufficient optimality conditions for structured nonlinear programs in infinite-dimensional function spaces, in Mathematical Programming with Data Perturbations, Lecture Notes in Pure and Appl. Math. 195, Marcel Dekker, New York, 1998, pp. 83-107.

[13] H. Goldberg And F. TröLtzSCH, Second-order sufficient optimality conditions for a class of nonlinear parabolic boundary control problems, SIAM J. Control Optim., 31 (1993), pp. $1007-1025$.

[14] E. Hewitt And K. Stromberg, Real and Abstract Analysis, Springer-Verlag, Berlin, Heidelberg, New York, 1965.

[15] A. D. Ioffe, Necessary and sufficient conditions for a local minimum 3: Second order conditions and augmented duality, SIAM J. Control Optim., 17 (1979), pp. 266-288.

[16] X. Li and J. Yong, Optimal Control Theory for Infinite Dimensional Systems, Birkhäuser Boston, Boston, 1995.

[17] M. Mateos, Problemas de control óptimo gobernados por ecuaciones semilineales con restricciones de tipo integral sobre el gradiente del estado, Ph.D. thesis, University of Cantabria, Santander, Spain, 2000.

[18] H. MAURER, First and second order sufficient optimality conditions in mathematical programming and optimal control, Math. Programming Study, 14 (1981), pp. 163-177.

[19] C. B. Morrey, Jr., Multiple integrals in the calculus of variations, Springer-Verlag, New York, 1966.

[20] J. RAymond AND F. Tröltzsch, Second order sufficient optimality conditions for nonlinear parabolic control problems with state-constraints, Discrete Contin. Dynam. Systems, 6 (2000), pp. 431-450.

[21] G. M. Troianiello, Elliptic Differential Equations and Obstacle Problems, Plenum Press, New York, 1987. 